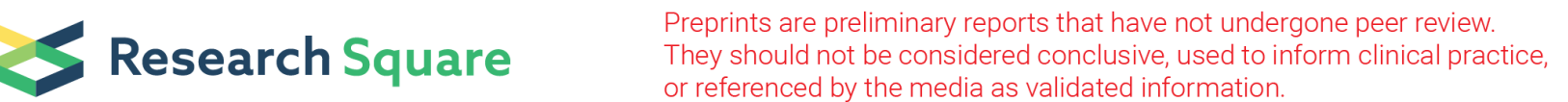

\section{Mettl14 Mediates the Inflammatory Response of Macrophages in Atherosclerosis Through the NF- KB/IL-6 Signaling Pathway}

\section{Yang Zheng}

Harbin Medical University Second Affiliated Hospital Department of Cardiology

\section{Yunqi Li}

Harbin Medical University Second Affiliated Hospital Department of Cardiology

\section{Xianwen Ran}

Harbin Medical University Second Affiliated Hospital Department of Cardiology

\section{Di Wang}

Harbin Medical University Second Affiliated Hospital Department of Cardiology

\section{Xianghui Zheng}

Harbin Medical University Second Affiliated Hospital Department of Cardiology

\section{Maomao Zhang}

Harbin Medical University Second Affiliated Hospital Department of Cardiology

\section{Jian Wu ( $\nabla$ wujian780805@163.com )}

Second Affiliated Hospital of Harbin Medical University https://orcid.org/0000-0002-1192-321X

\section{Yong Sun}

Harbin Medical University Institute of Biological Information Science and Technology

Bo Yu

Harbin Medical University Second Affiliated Hospital Department of Cardiology

\section{Research Article}

Keywords: Atherosclerosis, m6A modification, Mettl14, macrophage, inflammatory response

Posted Date: November 30th, 2021

DOI: https://doi.org/10.21203/rs.3.rs-1070642/v1

License: (c) (i) This work is licensed under a Creative Commons Attribution 4.0 International License. Read Full License 


\section{Abstract}

The inflammatory response of macrophages has been reported to play a critical role in atherosclerosis. The inflammatory state of macrophages is modified by epigenetic reprogramming. $\mathrm{m}^{6} \mathrm{~A}$ RNA methylation is an epigenetic modification of RNAs. However, little is known about the potential roles and underlying mechanisms of $\mathrm{m}^{6} \mathrm{~A}$ modification in macrophage inflammation. Herein, we showed that the expression of the $\mathrm{m}^{6} \mathrm{~A}$ modification "writer" Mettl14 was increased in coronary heart disease and LPS-stimulated THP-1 cells. Knockdown of Mettl14 promoted M2 polarization of macrophages, inhibited foam cell formation and decreased migration. Mechanistically, the expression of Myd88 and IL- 6 was decreased in Mettl14 knockdown cells. Through $\mathrm{m}^{6} \mathrm{~A}$ modification, Mettl14 regulated the stability of Myd88 mRNA. Furthermore, Myd88 affected the transcription of IL- 6 via the distribution of p65 in nuclei rather than directly regulating the expression of IL- 6 through $\mathrm{m}^{6} \mathrm{~A}$ modification. In vivo, Mett|14 gene knockout significantly reduced the inflammatory response of macrophages and the development of atherosclerotic plaques. Taken together, our data demonstrate that Mettl14 plays a vital role in macrophage inflammation in atherosclerosis via the NF-KB/IL- 6 signaling pathway, suggesting that Mett114 may be a promising therapeutic target for the clinical treatment of atherosclerosis.

\section{Introduction}

Monocytes and macrophages are regarded as the main actors in atherosclerosis because of their ubiquitous involvement during all stages of atherosclerosis[4, 8]. Considerable amounts of evidence support that atherosclerosis is a chronic inflammatory disease starting with the entry of monocytes from peripheral blood into the endothelium: rolling, adhesion, activation and migration. Then, monocytes differentiate into macrophages[5,27]. Depending on the markers, production of specific factors and biological functions of the macrophages, macrophages can be divided into two major subtypes: classically activated macrophages (M1) and alternatively activated macrophages (M2). High levels of the glycoprotein Ly6C (Ly6C $\left.{ }^{\text {high }}\right)$ monocytes in mice, known as CD14 (CD14 ${ }^{++}$CD16 $)$in humans, differentiate into M1 macrophages. However, Ly6 $6 \mathrm{C}^{\text {low }}$ monocytes (CD14 ${ }^{+/ l o w} \mathrm{CD} 16^{+}$in humans) differentiate into M2 macrophages[12,38]. M1 macrophages initiate and sustain inflammation, producing inflammatory cytokines (TNF- $\alpha$, IL-1 $\beta$ and IL-6) and leading to foam cell formation. M2 macrophages counteract inflammation and secrete anti-inflammatory cytokines (IL-10 and TGF- $\beta$ ) $[5,25]$. The biological characteristics of macrophages in atherosclerotic plaques determine the size, composition and stability of the lesion[22]. Recent studies suggest that a substantial proportion of patients retain the progression of the plaque despite achieving guideline-directed therapeutic targets caused by inflammation[31, 35]. Regulating the inflammatory state and function of macrophages raises hope for atherosclerosis regression.

Macrophages are associated with epigenetic reprogramming and are modified by epigenetic enzymes[20]. $\mathrm{N}^{6}$-methyladenosine $\left(\mathrm{m}^{6} \mathrm{~A}\right)$ RNA methylation is a posttranscriptional epigenetic modification of eukaryotic RNAs, including coding and noncoding RNAs[9]. The $\mathrm{m}^{6} \mathrm{~A}$ modification 
process is jointly regulated by three types of enzymes: "writers" (methyltransferases), "erasers" (demethylases) and "readers" ( $\mathrm{m}^{6} \mathrm{~A}$-related binding proteins)[36]. Previous research has established that $\mathrm{m}^{6} \mathrm{~A}$ modification is associated with RNA metabolism, including changes in RNA structure, splicing, export and translation[16]. Numerous studies have demonstrated that regulators of $\mathrm{m}^{6} \mathrm{~A}$ methylation are involved in multiple physiological and pathological processes, such as immune system development, human cancers and cardiovascular disease[16].

However, our understanding of the regulation of $\mathrm{m}^{6} \mathrm{~A}$ modification in atherosclerosis is still in its infancy. Guo et al. showed that IFN regulatory factor- 1 induced macrophage pyroptosis by Mettl3-mediated $\mathrm{m}^{6} \mathrm{~A}$ modification of circ_0029589 in atherosclerosis[13]. Zhang et al. demonstrated that Mettl14 increased the $\mathrm{m}^{6} \mathrm{~A}$ modification of pri-miR-19a to promote endothelial cell proliferation and invasion in vitro[44]. Similar studies have reported that Mettl14 regulates endothelial inflammation and that Mett|14 knockdown reduces the development of atherosclerotic plaques[15]. However, the amount of published data on the $\mathrm{m}^{6} \mathrm{~A}$ modification of macrophages in atherosclerosis is limited.

Here, we found that the Mettl14 "writer" can regulate the inflammatory state of macrophages in atherosclerosis. Knockdown of Mettl14 promoted M2 polarization and inhibited foam cell formation. Mechanistically, RNA sequencing (RNA-seq) analysis revealed that Mett|14 regulated the expression of Myd88 through $\mathrm{m}^{6} \mathrm{~A}$ modification. Myd88 affected the transcription of IL- 6 through p 65 by regulating the distribution of p65 in nuclei. In vivo, Mettl14 gene knockout in mice significantly reduced the development of atherosclerosis by decreasing the inflammatory response of macrophages. Collectively, our study offers important insights into the $\mathrm{m}^{6} \mathrm{~A}$ modification in atherosclerosis and highlights a potential target for treatment.

\section{Materials And Methods}

\section{Study population and ethics statement}

A total of 54 participants were selected for our study from the Second Affiliated Hospital of Harbin Medical University between December 2020 and June 2021: 9 controls, 18 patients with ST-segment elevation myocardial infarction (STEMI), 11 patients with non-ST-segment elevation myocardial infarction (NSTEMI) and 17 patients with unstable angina (UA). Inclusion and exclusion criteria were previously described in detail[23]. Briefly, patients with UA, STEMI and NSTEMI were enrolled according to the guidelines[39]. All participants or their families provided informed consent for inclusion before participation in the study, conforming to the Declaration of Helsinki. The current study was approved by the Ethics Committee of the Second Affiliated Hospital of Harbin Medical University, China (KY2020-156).

\section{Animal and atherosclerosis induction}

Mett|14 heterozygous mice (Mett|14 ${ }^{+/}$) were established from C57/BL6 mice by Cyagen Biosciences, Inc. (Suzhou, Jiangsu, China), using CRISPR/Cas9-based targeting and homology-directed repair. C57/BL6 
and $\mathrm{APOE}^{-/-}$mice were purchased from Beijing Vital River Laboratory Animal Technology (Beijing, China). Mettl14 ${ }^{+/-} \mathrm{APOE}^{-/-}$mice were generated by breeding Mettl144 ${ }^{+/-}$mice with $\mathrm{APOE}^{-/-}$mice. Eight- to 10-weekold male $\mathrm{APOE}^{-/-}$(WT) mice and Mettl14 ${ }^{+/-} \mathrm{APOE}^{-/-}$(KO) mice were fed a high-cholesterol diet (D12108C, Opensource diets) for 12 weeks. Then, the mice were euthanized for further analysis. All mice were housed under specific pathogen-free (SPF) conditions with controlled temperature and a 12-hour light/dark cycle at the Second Affiliated Hospital of Harbin Medical University. All experimental protocols were approved by the Institutional Animal Care and Use Committee at the Second Affiliated Hospital of Harbin Medical University (sydwgzr2020-095). This study was conducted in accordance with the Guide for the Care of Use of Laboratory Animals (Institute of Laboratory Animal Resources/National Institutes of Health, Bethesda, MD, USA).

\section{Peripheral blood mononuclear cells isolated}

Overnight fasting blood samples were collected by venipuncture from all participants when they were hospitalized on the first day. The peripheral blood of mice was collected when the mice were fed a highcholesterol diet for 12 weeks. Peripheral blood mononuclear cells (PBMCs) of humans and mice were isolated using the density gradient technique (TBD; Tianjin, China). Human PBMCs were lysed with TRIzol reagent (Invitrogen, Carlsbad, USA) for RNA extraction. The PBMCs of mice were further analyzed via flow cytometry.

\section{RNA extraction and qRT-PCR}

Total RNA from PBMCs of patients and THP-1 cells was extracted using TRIzol reagent (Invitrogen, Carlsbad, USA) and converted to complementary DNA by a Transcriptor First Strand cDNA Synthesis Kit (Roche, Basel, Switzerland). qRT-PCR was performed with a Transcriptor First Strand cDNA Synthesis Kit (Roche, Basel, Switzerland). The settings were as follows: 40 cycles of 10 seconds at $95^{\circ} \mathrm{C}, 30$ seconds at $60^{\circ} \mathrm{C}$, and 30 seconds at $72^{\circ} \mathrm{C}$. All relative mRNA expression levels were analyzed using the $2^{-\Delta \Delta \mathrm{Ct}}$ method. The primers used are listed in Table S1.

\section{Dot-blot assays}

Total RNA from PBMCs of patients and THP-1 cells was extracted using TRIzol reagent (Invitrogen, Carlsbad, USA). The RNAs (200, 100 and $50 \mathrm{ng}$ ) were double diluted, denatured by heating at $95^{\circ} \mathrm{C}$ for 5 min and chilled on ice immediately. The RNAs were then spotted onto nitrocellulose membranes (Solarbio, Beijing, China). Then, the membranes were ultraviolet (UV) crosslinked, blocked and incubated with an m6A-specific antibody (Synaptic Systems, Gottingen, Germany). The other membrane was stained with methylene blue as a loading control.

\section{Culture and transfection of THP-1 cells}

The human macrophage cell line THP-1 was purchased from the American Type Culture Collection. The cells were cultured in RPMI 1640 medium (Gibco, Thermo Fisher Scientific, Waltham, MA, USA) consisting 
of $10 \% \mathrm{FBS}$ (Biological Industries, Israel) at $37^{\circ} \mathrm{C}$ with $5 \% \mathrm{CO}_{2}$.

The siRNAs for siMettl14 and siMyd88 and the plasmid DNA were purchased from GenePharma (Shanghai, China). All the sequences of the siRNAs are provided in Table S1. The siRNAs were transfected into cells by Lipofectamine RNAimax (Invitrogen, Thermo Fisher Scientific, Waltham, MA, USA) for $48 \mathrm{~h}$. The plasmid DNAs were then transfected into cells by Lipofectamine 3000 (Invitrogen, Thermo Fisher Scientific, Waltham, MA, USA) for $48 \mathrm{~h}$. Then, the media were removed and replaced with fresh media. The cells treated with LPS ( $500 \mathrm{ng} / \mathrm{ml}$; Sigma-Aldrich, St. Louis, MO, USA) for $24 \mathrm{~h}$.

\section{Western blot and antibodies}

Proteins were isolated from THP-1 cells in RIPA buffer (Beyotime, Shanghai, China) together with a 1\% cocktail of protease (Roche, Basel, Switzerland) and 1 mM PMSF (Beyotime, Shanghai, China). Protein quantification was performed using a BCA protein detection kit (Beyotime, Shanghai, China). Protein lysates were separated by $8 \%-12.5 \%$ SDS-PAGE and transferred onto $0.22 \mu \mathrm{m}$ Immobilon-NC gels (Merck Millipore, Billerica, MA, USA). The primary antibodies used were anti-Mett114, anti-p65, anti-p-p65, anti-Ikb $a$, anti-ABCA1, anti-ABCG1, anti-PPAR-y, anti-LXR-a, anti-Myd88, anti-IL-6, and anti- $\beta$-actin, anti-GAPDH. The membranes were blocked in blocking buffer and then incubated with the primary antibodies overnight. The protein bands were visualized using chemiluminescence in a Tanon 5100 system (Tanon, Shanghai, China).

\section{Flow cytometry analysis}

THP-1 cells were treated with LPS $(500 \mathrm{ng} / \mathrm{ml})$ after transfection. The positive control groups for M1 and M2 were treated with LPS $(500 \mathrm{ng} / \mathrm{ml})$ or IL-4 $(20 \mathrm{ng} / \mathrm{ml})$, respectively. The cells were harvested and stained with PE-CD86 and APC-CD163 antibodies (BioLegend, San Diego, CA, USA) for 30 min at $4^{\circ} \mathrm{C}$ in the dark. Then, the cells were washed, fixed and permeabilized using Fix/Perm buffer (Invitrogen, Thermo Fisher Scientific, Waltham, MA, USA). FITC-CD68 (BioLegend, San Diego, CA, USA) was added to the cells for $30 \mathrm{~min}$ at room temperature. The PMBCs of mice were stained with FITC-Ly- $6 \mathrm{C}$ and PE-CX3CR1 (BioLegend, San Diego, CA, USA) for $30 \mathrm{~min}$ at $4^{\circ} \mathrm{C}$ in the dark. The data were analyzed using FACSDiva version 6.1.3 (BD Biosciences, San Jose, CA, USA) and FolwJo_V10 (TreeStar, Ashland, OR, USA).

\section{Intracellular cholesterol assessment}

THP-1 cells were treated with $50 \mu \mathrm{g} / \mathrm{ml}$ ox-LDL (YB-002, Yiyuan Biotechnologies, Guangzhou, China) after transfection for $24 \mathrm{~h}$. Then, the cells were washed with PBS and fixed with $4 \%$ paraformaldehyde for 20 min. Afterward, the cells were washed with $60 \%$ isopropanol and stained with an oil red 0 staining kit (cultured cells; Solarbio, Beijing, China).

\section{Wound healing}

After stimulation with LPS for $24 \mathrm{~h}$, the cell monolayer was scratched with a sterile pipette tip and washed with PBS. The scratch wound areas were observed at 0 and $12 \mathrm{~h}$. 


\section{RNA-seq and bioinformatics analysis}

RNA from the siM14 and NC groups was isolated using TRIzol reagent. RNA-seq and data analysis were carried out by Sangon Biotech (Shanghai, China). The cDNA library was constructed using a TruSeq PE Cluster Kit v4-cBot-HS (Illumina, USA). Sequencing was performed on a MGISEQ-2000 platform. The RNAseq reads were mapped by the human reference genome (hg19) from the NCBI using HISAT2. The differentially expressed genes (DEGs) were filtered based on $P$ values $<0.05$ and fold-changes $>1.5$. G0 enrichment and KEGG pathway enrichment analyses of DEGs were performed by using DAVID Bioinformatics Resources 6.8 (https://david.ncifcrf.gov/).

\section{Immunofluorescence}

The cells were fixed with $4 \%$ paraformaldehyde for $20 \mathrm{~min}$. The tissue was cut into $7 \mu \mathrm{m}$ thin slices and fixed in acetone. Then, the cells or cryosections were immersed in Triton X-100 (Biosharp, Hefei, China) for 30 min followed by supplementation with 10\% normal goat serum (Solarbio, Beijing, China) for 30 min. The cells or cryosections were incubated overnight with primary antibodies. Secondary antibodies were added to the cells or cryosections and then labeled with DAPI. The images were captured using a confocal laser scanning microscope (Zeiss LSM 700).

\section{mRNA decay analysis}

THP- 1 cells were treated with actinomycin $D$ at a final concentration of $5 \mu \mathrm{g} / \mathrm{ml}$ for 5 or $10 \mathrm{~h}$. Total RNA was extracted at the indicated time points for reverse transcription and qRT-PCR. The mRNA decay rate was normalized to that at $0 \mathrm{~h}$.

\section{RNA-binding protein immunoprecipitation assays}

RIP assays were performed using an RNA Immunoprecipitation Kit (Geneseed Biotech, Guangzhou, China) according to the manufacturer's instructions. In brief, the cells were harvested in RIP buffer on ice for $10 \mathrm{~min}$. Then, $100 \mu \mathrm{L}$ RIP lysis was used as input. Then, the cell lysates were incubated with $5 \mu \mathrm{g}$ anti$\mathrm{m}^{6} \mathrm{~A}$ antibody or control IgG containing protein $\mathrm{A} / \mathrm{G}$-agarose beads. After rotation at $4^{\circ} \mathrm{C}$ for $2 \mathrm{~h}$, the beads were washed. The immunoprecipitated and input RNAs were isolated and subjected to RIP-qPCR analysis. The PCR primers used are listed in Table S1.

\section{Chromatin immunoprecipitation (CHIP) assays}

ChIP assays were performed using a SimpleChIP Enzymatic Chromatin IP Kit (Cell Signaling Technology, Danvers, MA, USA). The cells were crosslinked with $1 \%$ formaldehyde for $10 \mathrm{~min}$ and then sonicated in lysis buffer. Ten microliters of the lysate was used as an input. The remaining lysate was subjected to a ChIP assay using p65 or IgG antibodies. The PCR primers used are provided in Table S1.

\section{Pathological staining}


The hearts from the mice were perfused with PBS and immediately embedded in Tissue OCT-Freeze Medium (Tissue-Tek, Sakura, Torrance, CA). Serial cryosections $(7 \mu \mathrm{m})$ were made using a cryostat. The serial cryosections from the caudal aortic sinus to the proximal aorta were stained with hematoxylin and eosin (HE) to visualize the plaques, oil red $\mathrm{O}$ to evaluate the lipid content, and Masson trichrome to analyze lesion size, collagen content, fibrous cap thickness and necrotic core formation. The HE staining kit, oil red $\mathrm{O}$ staining kit and Masson trichrome staining kit were purchased from Solarbio (Beijing, China).

\section{ELISA analysis}

Plasma was collected from the mice. Then, the levels of IL-6 were assessed with ELISA kits (Lianke Biotech, Hangzhou, China) according to the manufacturer's instructions. The absorbance was measured using a microplate reader (Tecan Infinite M200).

\section{Serum lipid determination}

Sera were collected from the mice. Serum lipids, including triglycerides, total cholesterol, LDLs and HDLs, were determined based on commercial kits (Jiancheng, Nanjing, China) using a microplate reader (Tecan Infinite M200).

\section{Statistical analysis}

The results are presented as the means \pm SDs. The statistical analyses were performed using GraphPad Prism version 9.1.0 or SPSS 23.0 software (IBM). Two-group comparisons were performed by unpaired two-tailed Student's t-test, and three or more group comparisons were performed by ordinary one-way analysis of variance (ANOVA). $P$ values $<0.05$ were considered statistically significant. All experiments were performed at least three times.

\section{Results}

\section{Mett|14 is upregulated in coronary heart disease patients and macrophages}

The role of $\mathrm{m}^{6} \mathrm{~A}$ in PBMCs of patients with coronary heart disease (CHD) was evaluated using an $\mathrm{m}^{6} \mathrm{~A}$ mRNA dot blot assay. We divided patients with CHD into the UA group, STEMI group and NSTEMI group. The levels of $\mathrm{m}^{6} \mathrm{~A}$ modification, including UA, STEMI and NSTEMI, were significantly increased in CHD (Fig. 1a). Given that the $\mathrm{m}^{6} \mathrm{~A}$ modification is primarily catalyzed by methyltransferase (writers) and demethylase (erasers), we hypothesized that the abnormal $\mathrm{m}^{6} \mathrm{~A}$ modification in $\mathrm{CHD}$ was caused by the dysregulation of $\mathrm{m}^{6} \mathrm{~A}$ writers and erasers. Then, we measured the expression of writers (Mettl3, Mettl14, Mettl16 and WTAP) and erasers (FTO and ALKBH5) in the PBMCs of CHD patients. Interestingly, the expression of Mett|3 and Mett|14 was significantly increased in CHD patients, including those in the UA group, STEMI group and NSTEMI group (Fig. 1b,c). The level of WTAP was increased in the UA group and NSTEMI group but did not change in STEMI group (Fig. 1e). FTO was upregulated in the UA group but did not change in the STEMI or NSTEMI group (Fig. 1f). The level of ALKBH5 was decreased in the STEMI 
and NSTEMI groups but did not change in the UA group (Fig. 1g). In addition, Mettl16 did not change significantly in CHD patients (Fig. 1d). Consistent with the results in $\mathrm{CHD}$ patients, $\mathrm{m}^{6} \mathrm{~A}$ modification in LPS-stimulated THP-1 cells (a human monocyte cell line) increased (Fig. 2a). Moreover, the expression of Mett|3, Mett114, Mett|16 and WTAP was increased in LPS-stimulated THP-1 cells, and ALKBH5 and FTO expression decreased in LPS-stimulated THP-1 cells (Fig. 2b-c, Fig. S1a-e). In particular, the expression level of Mett114 was highest in LPS-stimulated THP-1 cells. Taken together, these results prompted us to explore the consequences of increased Mettl14 in macrophages caused by atherosclerosis.

\section{Mett|14 knockdown conditioned macrophages toward M2 polarization and suppressed NF-KB signaling}

The $\mathrm{m}^{6} \mathrm{~A}$ writer Mettl14 plays a critical role in the regulation of $\mathrm{m}^{6} \mathrm{~A}$ modification in macrophages in atherosclerosis. Hence, we knocked down Mettl14 in THP-1 cells through two different siRNAs. qRT-PCR and Western blot analysis showed that both siM14-1 and siM14-2 caused a decrease in the expression of Mett14 (Fig. S1f-h). LPS- and IL-4-stimulated THP-1 cells were used as positive controls for M1 and M2 polarization, respectively (Fig. 2d, Fig. S1i-j). As shown in Fig. 2d and Fig. S1i-j, knockdown of Mettl14 significantly increased the proportion of $\mathrm{M} 2$ macrophages $\left(\mathrm{CD} 68^{+} \mathrm{CD} 163^{+}\right.$cells) and decreased the proportion of $\mathrm{M} 1$ macrophages (CD68 ${ }^{+} \mathrm{CD} 86^{+}$cells). Like flow cytometry, qRT-PCR confirmed that the mRNA expression of IL-1 $\beta$ and TNF-a was downregulated and that IL-10 and CD163 were upregulated in siM14-1- and siM14-2-treated THP-1 cells (Fig. 2e-h). Previous studies have demonstrated that the NF-KB pathway plays an important role in macrophage polarization by inducing the expression of inflammatory genes. Then, we investigated the NF-KB pathway using western blotting to evaluate whether the NF-kB pathway was involved in macrophage polarization. Knockdown of Mettl14 markedly reduced the phosphorylation of NF-KB-p65, but the level of p65 was not significantly changed (Fig. 2i, Fig. S1k-I). The expression of $\mathrm{IKBa}$ increased in M2 phenotype macrophage cells (Fig. 2i, Fig. S1 m). Taken together, these data suggested that knockdown of Mettl14 prevents the macrophage inflammatory response by promoting M2 polarization via the NF-KB pathway.

\section{Mett14 deficiency inhibited foam cell formation and macrophage migration}

In arteries, foam cell formation and macrophage migration play important roles in the development of atherosclerosis. We asked whether Mettl14 has a role in foam cell formation and macrophage migration. As shown in Fig. 3a and 3b, the siM14-1 and siM14-2 groups had much less lipid accumulation than the $\mathrm{NC}$ group stained with oil red $\mathrm{O}$ did. Then, we measured the expression level of lipid metabolism-related proteins. Several studies have shown that $A B C A 1$ and ABCG1 have an essential role in cholesterol efflux during foam cell formation and that PPAR- $y$ and LXR-a regulate ABCG1/ABCA1[6]. The expression of ABCA1, ABCG1, PPAR-y and LXR-a was increased in the Mettl14 knockdown group compared to the NC group (Fig. 3c-g), indicating that the protective function of Mettl14 in macrophages may be related to the PPARY-LXRa-ABCA1/ABCG1 pathway. Next, using a scratch test, we confirmed that Mettl14 affected migration. The results showed that knockdown of Mettl14 significantly decreased macrophage migration (Fig. 3h-i). Together, the above findings demonstrated that Mettl14 deficiency inhibited foam cell formation and macrophage migration. 


\section{Mettl14 modifies Myd88 mRNA stability by $\mathrm{m}^{6} \mathrm{~A}$ modification}

To determine Mettl14-regulated genes in macrophages, we used RNA-seq. For this, we used three samples of Mettl14 knockdown THP-1 cells and three samples of THP-1 cells treated with NC. The RNA-seq data showed that 150 mRNAs were upregulated and that 128 mRNAs were downregulated in the Mettl14 knockdown group compared with the NC group (Fig. 4a, Table S2). Gene Ontology (GO) analysis of these DEGs showed that they were involved mainly in the immune response, inflammatory response, chronic inflammatory response, positive regulation of interleukin-6 production and positive regulation of I-KB kinase/NF-KB signaling (Fig. 4b). The Kyoto Encyclopedia of Genes and Genomes (KEGG) pathway was mainly enriched in cytokine-cytokine receptor interaction, rheumatoid arthritis, pertussis, Toll-like receptor signaling pathway and inflammatory bowel disease (Fig. 4c). These data suggested that Mettl14 regulated the inflammatory response of macrophages.

Among the DEGs, Myd88 and interleukin-6 (IL-6) were very interesting because they play an important role in the inflammatory response of macrophages[1, 3, 7]. First, we detected whether the expression of Myd88 was regulated by Mettl14. qRT-PCR analysis revealed that the mRNA expression levels of Myd88 were markedly lower in Mettl14 knockdown THP-1 cells than in those in the NC group, whereas the mRNA expression of Myd88 was increased in the Mettl14 overexpression group (Fig. 4d). Moreover, using western blots and immunofluorescence staining, we obtained similar results of Myd88 protein expression (Fig. 4e-f, Fig. S2a). To determine the regulatory mechanism by which Mettl14 regulates Myd88, we examined the mRNA stability of Myd88. Similar to our hypothesis, the stability of Myd88 mRNA was decreased in the Mettl14 knockdown group (Fig. 4i). RNA immunoprecipitation (RIP) analysis revealed that knockdown of Mettl14 reduced the $\mathrm{m}^{6} \mathrm{~A}$ modification on site 4 of Myd88 mRNA (Fig. 4j). These results suggested that Mettl14 modifies Myd88 mRNA stability by $\mathrm{m}^{6} \mathrm{~A}$ modification.

\section{Mett|14 regulates the expression of IL-6 by affecting the nuclear distribution of p65 through Myd88}

IL-6 is a key inflammatory factor involved in the activation and regulation of macrophages[40]. To validate the RNA-seq results, we performed qRT-PCR to detect the expression of IL-6 mRNA. The level of IL- 6 was significantly decreased in the Mettl14 knockdown group, and the expression of IL- 6 was the opposite in the Mettl14 overexpression group (Fig. 4h). Next, we identified the mechanism by which MettI14 regulates IL- 6 . Unfortunately, the stability of IL- 6 mRNA and translation was not significantly affected by Mettl14 (Fig. 4g-k, Fig. S2b). Therefore, Mettl14 does not regulate IL-6 through $\mathrm{m}^{6} \mathrm{~A}$ modification. Therefore, how does Mettl14 regulate the expression of IL-6? We assumed that Mett|14 regulated IL-6 through the Myd88/NF-KB pathway according to the prediction of p65 binding to the promoter region of IL-6. Mettl14 did not affect the expression level of p65 (Fig. 2i). Immunofluorescence staining showed that knockdown of Mettl14 impeded p65 translocation from the cytosol to the nucleus (Fig. 4I). However, p65 translocation from the cytosol to the nucleus was facilitated in THP-1 cells treated with siM14 and overexpressing Myd88 (Fig. 4I). As expected, ChIP analysis revealed that knockdown of Mettl14 inhibited p65 binding to the IL-6 promoter (Fig. 4m, Fig. S2c). P65 binding to the IL-6 promoter 
was increased in cotransfected THP-1 cells. These results indicated that Mettl14 regulates its expression through the Myd88/NF-kB pathway.

\section{Knockdown of Mettl14 induces a low inflammatory response in macrophages through Myd88}

We showed that Mettl14 knockdown induced a low inflammatory response in macrophages (Fig. 2 and Fig. 3) and that Myd88 was regulated by Mettl14 (Fig. 4). We next examined whether the functions of Mettl14 are mediated by Myd88 in macrophages. For this, we conducted rescue experiments. We generated a Mett|14 expression vector, which was cotransfected with siMyd88. Overexpression of Mett|14 promoted M1 polarization rather than M2 polarization in THP-1 cells (Fig. 5a-c). As expected, siMyd88 reversed Mettl14 overexpression-induced M1 polarization (Fig. 5a-c). The expression level of p65 protein did not significantly change in the OE-M14 group or cotransfection group. The p-p65 protein level increased in Mettl14-overexpressing THP-1 cells, and its expression was decreased by siMyd88 (Fig. 5d, Fig. S3a-c). We found that the inflammatory factors IL-1 $\beta$ and TNF-a increased and that the antiinflammatory factors IL-10 and CD163 decreased in Mettl14-overexpressing cells (Fig. 5e-h). Moreover, the levels of inflammatory factors and anti-inflammatory factors were reversed in cells cotransfected with siMyd88. Furthermore, Mettl14 overexpression increased foam cell formation and decreased the abundance of lipid metabolism-related proteins (Fig. 5i-k). However, foam cell formation decreased in response to siMyd88, and lipid metabolism-related proteins increased (Fig. 5i-k, Fig. S2d-g). The migration of macrophages had similar results. Overexpression of Mett114 promoted the migration of macrophages (Fig. 5I-m). However, siMyd88 reversed OE-M14-induced migration. Collectively, these results suggested that Mettl14 regulates the functions of macrophages through Myd88.

\section{Mett|14 deficiency attenuates atherosclerosis progression in vivo}

Given that Mettl14 regulates the functions of macrophages in vitro, we further assessed the effect of Mett|14 on atherosclerosis progression in vivo. Previous studies have demonstrated that Mettl14 gene knockout causes embryo death. Mettl14 heterozygous knockout mice were used for research[15]. To do this, age- and weight-matched male $\mathrm{APOE}^{-/-}(\mathrm{WT})$ mice and Mettl1 $14^{+/-} \mathrm{APOE}^{-/-}(\mathrm{KO})$ mice were fed a western diet for three months. After three months, the aortas and peripheral blood of these mice were dissected. Compared to the WT mice, the KO mice did not exhibit changes in the plasma lipid profiles (Fig. S4a-c). As shown in Fig. 6a, images of aortic arches suggested that $\mathrm{KO}$ mice were significantly protected from atherosclerosis. $\mathrm{HE}$ staining of the aortic roots revealed that the lesion areas were smaller in $\mathrm{KO}$ mice than in WT mice (Fig. 6b, Fig. S4d). The extent of proximal aortic atherosclerosis was reduced in KO mice using oil red $\mathrm{O}$ (Fig. 6c, Fig.S4e). These results suggested that Mettl14 knockout can significantly reduce atherosclerosis.

Next, using Masson trichrome staining, we examined the characteristics of plaque stabilization between WT and KO mice. Compared to the WT mice, the KO mice had an increased collagen content in the proximal aorta (Fig. 6d, Fig. S4f). In addition, the thickness of the fibrous cap was greater in the lesions of KO mice than in those of WT mice (Fig. 6d, Fig. S4g). Importantly, the necrotic area was decreased in KO 
mice vs. WT mice (Fig. 6d, Fig. S4h). Taken together, these data showed that Mettl14 deficiency suppressed the characteristics of vulnerable plaque formation.

\section{Mett|14 regulates the function of macrophages via Myd88/IL-6 in vivo}

To verify the function and mechanism of macrophages regulated by Mettl14 in vivo, we analyzed the subtypes of monocytes in the peripheral blood of the mice. The subtypes of monocytes include proinflammatory Ly $6 C^{\text {high }} \mathrm{CX} 3 \mathrm{CR} 1^{\text {low }}$ monocytes and anti-inflammatory Ly $6 \mathrm{C}^{\text {low }} \mathrm{CX} 3 \mathrm{CR} 1^{\text {high }}$ monocytes. We found that the subpopulations of Ly $6 \mathrm{C}^{\text {high }} \mathrm{CX} 3 \mathrm{CR} 1^{\text {low }}$ monocytes were lower in KO mice than in WT mice and that $\mathrm{Ly}_{6} \mathrm{C}^{\mathrm{low}} \mathrm{CX} 3 \mathrm{CR} 1^{\text {high }}$ monocytes were higher (Fig. 6e-g). These data indicate that Mettl14 deficiency reprograms monocytes/macrophages to anti-inflammatory effects. Then, we detected the expression of Myd88 in plaque macrophages. Fig. $6 i$ shows that the expression of Myd88 significantly decreased in plaque macrophages in KO mice. Surprisingly, we also found that the plasma level of IL-6 was reduced in $\mathrm{KO}$ mice (Fig. $6 \mathrm{~h}$ ). In conclusion, the results suggest that Mettl14 can regulate the function of macrophages in atherosclerosis via Myd88/IL-6 in vivo.

\section{Discussion}

The presence of macrophages is a common condition that has considerable impact on all stages of atherosclerosis $[4,8]$. The functions of macrophages are mainly regulated by epigenetic reprogramming[20]. Recent evidence suggests that epigenetic modifications can be grouped into three categories: epigenetic triad, DNA methylation, and histone modification and nucleosome positioning[30]. In recent years, the mechanism of epigenetic modifications has been updated with the development of specific methylated RNA immunoprecipitation and next-generation sequencing[28]. $M^{6} A$ is the most common epitranscriptomic modification of mRNA from yeast, plants, files, humans and other mammals[45]. $\mathrm{M}^{6} \mathrm{~A}$ methylation marks are dynamic and reversible. Briefly, $\mathrm{m}^{6} \mathrm{~A}$ methylation occurs at the consensus sequence $\mathrm{RRACH}(\mathrm{R}=\mathrm{G}$ or $\mathrm{A} ; \mathrm{H}=\mathrm{A}, \mathrm{C}$ or $\mathrm{U}$ ) through methyltransferases (writers) and demethylases (erasers). Then, the $\mathrm{m}^{6} \mathrm{~A}$-related binding proteins (readers) selectively bind the site of $\mathrm{m}^{6} \mathrm{~A}$ modification. After this regulatory event, RNAs are cleaved, stable, degraded and translated[18, 21, 34]. Several studies have demonstrated a link between $\mathrm{m}^{6} \mathrm{~A}$ modification and human diseases[34].

Zhang et al. demonstrated that $\mathrm{m}^{6} \mathrm{~A}$ modification and Mettl14 were significantly increased in the atherosclerotic vascular endothelial cells of patients with carotid stenosis[44]. Guo et al. showed that the expression of Mettl3 was significantly elevated in macrophages in patients with acute coronary syndrome[13]. Another study showed opposite results, which may be caused by different detection methods for $\mathrm{m}^{6} \mathrm{~A}$ modification. The $\mathrm{m}^{6} \mathrm{~A}$ levels were significantly decreased in peripheral blood mononuclear cells, as detected by colorimetry. These three studies suggest that $\mathrm{m}^{6} \mathrm{~A}$ modification plays an important role in atherosclerosis. Here, we observed that the levels of $\mathrm{m}^{6} \mathrm{~A}$ modification and Mettl14 were increased in the peripheral blood mononuclear cells of patients with coronary heart disease and LPS-stimulated THP-1 cells. It is possible, therefore, that Mettl14 has a pivotal role in macrophages in 
atherosclerosis. Existing studies recognize the critical role played by $\mathrm{m}^{6} \mathrm{~A}$ modification in the immune response of macrophages. An earlier study showed that the reader YTHDF2 was upregulated after LPS stimulation and that YTHDF2 knockdown promoted the inflammatory response in LPS-stimulated macrophages[43]. Subsequent studies confirmed that $\mathrm{m}^{6} \mathrm{~A}$ modification regulated $\mathrm{M} 1 / \mathrm{M} 2$ polarization and cholesterol efflux[24, 29, 41, 42]. However, the function of Mettl14 in macrophages in atherosclerosis has not been reported. In this study, we first reported that Mettl14 regulated the inflammatory state of macrophages in atherosclerosis. Knockdown of Mettl14 in macrophages promotes M2 polarization. Moreover, foam cell formation and migration were inhibited in Mettl14 knockdown macrophages. Atherosclerotic plaques and the inflammatory response were significantly reduced in Mettl14 knockout mice. These data provide evidence that Mett114 plays an essential role in the regulation of macrophages in atherosclerosis.

The mechanisms of $\mathrm{m}^{6} \mathrm{~A}$ modification are diverse, including the fold, stability, degradation and cellular interactions of the modified RNA[28]. Jian et al. demonstrated that Mettl14 enhances the transcription factor FOXO1 by increasing its translation, not RNA stabilization, in endothelial inflammation[15]. In bacterial infection, Mettl14 depletion blocked $\mathrm{m}^{6} \mathrm{~A}$ methylation of SOCS1, diminishing its RNA stability[10]. In addition, Mettl14 forms a complex with Mett|3, called the Mett|3/Mett114 complex, modifying nascent transcripts whose translation is enhanced[14]. We performed RNA-seq of Mettl14knockdown macrophages to explore the regulatory mechanism of Mettl14. The DEGs regulated by Mettl14 were enriched in the inflammatory response, indicating that Mettl14 plays an important role in the inflammatory response in macrophages. We found two interesting DEGs, those encoding Myd88 and IL-6. qRT-PCR further confirmed that the expression of Myd88 and IL-6 was consistent with the RNA-seq results. Most Toll-like receptors (TLRs) and several cytokine receptors signal through Myd88 to initiate a rapid immune response when alarmins stimulate macrophages[7]. In addition, Myd88 knockout mice showed smaller atherosclerotic plaques and less macrophage activation, lipid accumulation and foam cell formation[3, 26, 37]. Myd88 plays a central role in the inflammatory response of macrophages. Interestingly, Myd88 was a target gene of Mett|14 in our study. Through what mechanism does Mett|14 regulate Myd88? We measured Myd88 mRNA stability in Mettl14 knockdown THP-1 cells. As expected, the stability and $\mathrm{m}^{6} \mathrm{~A}$ modification of Myd88 mRNA were decreased in Mettl14 knockdown cells. Thus, Mettl14 regulates Myd88 through $\mathrm{m}^{6} \mathrm{~A}$ modification.

IL- 6 is a major contributor to the development of atherosclerosis. The aortas of atherosclerotic mice and rats had higher levels of macrophage-attracting IL- 6 than did those of the control, and the expression level of IL- 6 was higher with aging[2, 11]. The mice were given an anti-mouse IL- 6 receptor antibody, and the atherosclerotic lesion size and inflammation were reduced[1]. In clinical trials, the first cytokine inhibition, IL-1 $\beta$ inhibition, was used for atherosclerosis treatment and prevention and achieved results. The results showed that IL- 6 was the central signaling cytokine of IL-1 $\beta$ inhibition, in turn reducing the inflammatory response[33]. In a recent RESCUE trial, the IL-6 ligand monoclonal antibody ziltivekimab was highly effective at reducing the inflammatory response and atherosclerotic biomarkers, suggesting that IL-6 has become a new therapeutic target for atherosclerosis[32]. In our study, IL-6 was the target of

Page $12 / 27$ 
Mett|14. The expression of IL-6 was decreased in the Mettl14 knockdown, but the stability and translation of IL-6 mRNA did not significantly change in the Mettl14 knockdown. Mettl14 may therefore not depend on $\mathrm{m}^{6} \mathrm{~A}$ modification to regulate the expression of IL-6. On the basis of our results, we found that Mettl14 regulates the expression of Myd88 via $\mathrm{m}^{6} \mathrm{~A}$ modification, which is the upstream regulation of the NF-KB pathway. Several studies have demonstrated that NF-KB/p65 can regulate the transcription of IL-6[17, 19]. Therefore, we hypothesized that Mett114 regulates IL-6 through the Myd88/NF-KB pathway. As expected, Mettl14 regulated the distribution of p65 in nuclei, which regulates the transcription of IL-6, reflecting the mechanism of upstream regulation of IL-6 in macrophages.

In summary, our study explores the regulatory mechanisms of macrophage inflammation from a new epigenetic perspective (Fig. 7). Our findings confirmed that Mettl14 can decrease the inflammatory response of macrophages. In vitro, Mettl14 can reduce the stability of Myd88 mRNA via $\mathrm{m}^{6} \mathrm{~A}$ modification, thereby increasing M2 macrophage polarization and decreasing foam cell formation and migration of macrophages. Furthermore, we also demonstrated that Mettl14 regulates the transcription of IL-6 through the NF-KB/p65 pathway. In vivo, Mettl14 knockout in mice decreased atherosclerotic plaques and the inflammatory response. Our data indicate that Mettl14 may be a promising therapeutic target of macrophages in the treatment of atherosclerosis.

\section{Declarations}

\section{Funding}

This work was supported by the National Natural Science Foundation of China (Grant No.82002376, 81972143, 82072030 and 82172537), HMU Marshal Initiative Funding (Grant No.HMUMIF21021).

\section{Author contributions}

JW and $Y S$ conceived and designed the research. $Y Z, Y L, R X$ and DW performed the experiments. $Y Z$ and $\mathrm{XZ}$ performed the model of atherosclerosis. $\mathrm{YZ}, \mathrm{MZ}$ and $\mathrm{BY}$ analyzed the data. $\mathrm{YZ}$ wrote the manuscript.

\section{References}

1. Akita K, Isoda K, Sato-Okabayashi Y, Kadoguchi T, Kitamura K, Ohtomo F, Shimada K, Daida H (2017) An Interleukin-6 Receptor Antibody Suppresses Atherosclerosis in Atherogenic Mice. Front Cardiovasc Med 4:84 doi:10.3389/fcrm.2017.00084

2. Belmin J, Bernard C, Corman B, Merval R, Esposito B, Tedgui A (1995) Increased production of tumor necrosis factor and interleukin- 6 by arterial wall of aged rats. Am J Physiol 268:H2288-2293 doi:10.1152/ajpheart.1995.268.6.H2288 
3. Bjorkbacka H, Kunjathoor VV, Moore KJ, Koehn S, Ordija CM, Lee MA, Means T, Halmen K, Luster AD, Golenbock DT, Freeman MW (2004) Reduced atherosclerosis in MyD88-null mice links elevated serum cholesterol levels to activation of innate immunity signaling pathways. Nat Med 10:416-421 doi: $10.1038 / \mathrm{nm} 1008$

4. Checkouri E, Blanchard V, Meilhac O (2021) Macrophages in Atherosclerosis, First or Second Row Players? Biomedicines 9 doi:10.3390/biomedicines 9091214

5. Chinetti-Gbaguidi G, Colin S, Staels B (2015) Macrophage subsets in atherosclerosis. Nat Rev Cardiol 12:10-17 doi:10.1038/nrcardio.2014.173

6. Chistiakov DA, Melnichenko AA, Myasoedova VA, Grechko AV, Orekhov AN (2017) Mechanisms of foam cell formation in atherosclerosis. J Mol Med (Berl) 95:1153-1165 doi:10.1007/s00109-017-15758

7. Deguine J, Barton GM (2014) MyD88: a central player in innate immune signaling. F1000Prime Rep 6:97 doi:10.12703/P6-97

8. Dolfi B, Gallerand A, Haschemi A, Guinamard RR, Ivanov S (2021) Macrophage metabolic regulation in atherosclerotic plaque. Atherosclerosis 334:1-8 doi:10.1016/j.atherosclerosis.2021.08.010

9. Dong L, Cao Y, Hou Y, Liu G (2021) N(6) -methyladenosine RNA methylation: A novel regulator of the development and function of immune cells. J Cell Physiol doi:10.1002/jcp.30576

10. Du J, Liao W, Liu W, Deb DK, He L, Hsu PJ, Nguyen T, Zhang L, Bissonnette M, He C, Li YC (2020) N(6)-Adenosine Methylation of Socs1 mRNA Is Required to Sustain the Negative Feedback Control of Macrophage Activation. Dev Cell 55:737-753 e737 doi:10.1016/j.devcel.2020.10.023

11. Du W, Wong C, Song Y, Shen H, Mori D, Rotllan N, Price N, Dobrian AD, Meng H, Kleinstein SH, Fernandez-Hernando C, Goldstein DR (2016) Age-associated vascular inflammation promotes monocytosis during atherogenesis. Aging Cell 15:766-777 doi:10.1111/acel.12488

12. Geissmann F, Jung S, Littman DR (2003) Blood monocytes consist of two principal subsets with distinct migratory properties. Immunity 19:71-82 doi:10.1016/s1074-7613(03)00174-2

13. Guo M, Yan R, Ji Q, Yao H, Sun M, Duan L, Xue Z, Jia Y (2020) IFN regulatory Factor-1 induced macrophage pyroptosis by modulating m6A modification of circ_0029589 in patients with acute coronary syndrome. Int Immunopharmacol 86:106800 doi:10.1016/j.intimp.2020.106800

14. Ianniello Z, Sorci M, Ceci Ginistrelli L, laiza A, Marchioni M, Tito C, Capuano E, Masciarelli S, Ottone T, Attrotto C, Rizzo M, Franceschini L, de Pretis S, Voso MT, Pelizzola M, Fazi F, Fatica A (2021) New insight into the catalytic -dependent and -independent roles of METTL3 in sustaining aberrant translation in chronic myeloid leukemia. Cell Death Dis 12:870 doi:10.1038/s41419-021-04169-7 
15. Jian D, Wang Y, Jian L, Tang H, Rao L, Chen K, Jia Z, Zhang W, Liu Y, Chen X, Shen X, Gao C, Wang S, Li M (2020) METTL14 aggravates endothelial inflammation and atherosclerosis by increasing FOX01 N6-methyladeosine modifications. Theranostics 10:8939-8956 doi:10.7150/thno.45178

16. Jiang X, Liu B, Nie Z, Duan L, Xiong Q, Jin Z, Yang C, Chen Y (2021) The role of m6A modification in the biological functions and diseases. Signal Transduct Target Ther 6:74 doi:10.1038/s41392-02000450-x

17. Kawashima T, Murata K, Akira S, Tonozuka Y, Minoshima Y, Feng S, Kumagai H, Tsuruga H, Ikeda Y, Asano S, Nosaka T, Kitamura T (2001) STAT5 induces macrophage differentiation of M1 leukemia cells through activation of IL-6 production mediated by NF-kappaB p65. J Immunol 167:3652-3660 doi:10.4049/jimmunol.167.7.3652

18. Klungland A, Dahl JA (2014) Dynamic RNA modifications in disease. Curr Opin Genet Dev 26:4752 doi:10.1016/j.gde.2014.05.006

19. Kong HJ, Nam BH, Kim YO, Kim WJ, Cho HK, Lee CH, Lee SJ, Kim KK (2010) Characterization of the flounder IL-6 promoter and its regulation by the p 65 NF-kappaB subunit. Fish Shellfish Immunol 28:961-964 doi:10.1016/j.fsi.2010.01.014

20. Kuznetsova T, Prange KHM, Glass CK, de Winther MPJ (2020) Transcriptional and epigenetic regulation of macrophages in atherosclerosis. Nat Rev Cardiol 17:216-228 doi:10.1038/s41569-0190265-3

21. Lence T, Soller M, Roignant JY (2017) A fly view on the roles and mechanisms of the m(6)A mRNA modification and its players. RNA Biol 14:1232-1240 doi:10.1080/15476286.2017.1307484

22. Lin P, Ji HH, Li YJ, Guo SD (2021) Macrophage Plasticity and Atherosclerosis Therapy. Front Mol Biosci 8:679797 doi:10.3389/fmolb.2021.679797

23. Liu X, Li S, Yang Y, Sun Y, Yang Q, Gu N, Li J, Huang T, Liu Y, Dong H, Sun S, Fu G, Wu J, Yu B (2021) The IncRNA ANRIL regulates endothelial dysfunction by targeting the let-7b/TGF-betaR1 signalling pathway. J Cell Physiol 236:2058-2069 doi:10.1002/jcp.29993

24. Liu Y, Liu Z, Tang H, Shen Y, Gong Z, Xie N, Zhang X, Wang W, Kong W, Zhou Y, Fu Y (2019) The $\mathrm{N}(6)$-methyladenosine (m(6)A)-forming enzyme METTL3 facilitates M1 macrophage polarization through the methylation of STAT1 mRNA. Am J Physiol Cell Physiol 317:C762-C775 doi:10.1152/ajpcell.00212.2019

25. McArdle S, Buscher K, Ghosheh Y, Pramod AB, Miller J, Winkels H, Wolf D, Ley K (2019) Migratory and Dancing Macrophage Subsets in Atherosclerotic Lesions. Circ Res 125:1038-1051 doi:10.1161/CIRCRESAHA. 119.315175 
26. Michelsen KS, Wong MH, Shah PK, Zhang W, Yano J, Doherty TM, Akira S, Rajavashisth TB, Arditi M (2004) Lack of Toll-like receptor 4 or myeloid differentiation factor 88 reduces atherosclerosis and alters plaque phenotype in mice deficient in apolipoprotein E. Proc Natl Acad Sci U S A 101:1067910684 doi:10.1073/pnas.0403249101

27. Moore KJ, Sheedy FJ, Fisher EA (2013) Macrophages in atherosclerosis: a dynamic balance. Nat Rev Immunol 13:709-721 doi:10.1038/nri3520

28. Oerum S, Meynier V, Catala $M$, Tisne $C$ (2021) A comprehensive review of m6A/m6Am RNA methyltransferase structures. Nucleic Acids Res 49:7239-7255 doi:10.1093/nar/gkab378

29. Park MH, Jeong E, Choudhury M (2020) Mono-(2-Ethylhexyl)phthalate Regulates Cholesterol Efflux via MicroRNAs Regulated m(6)A RNA Methylation. Chem Res Toxicol 33:461-469 doi:10.1021/acs.chemrestox.9b00367

30. Portela A, Esteller M (2010) Epigenetic modifications and human disease. Nat Biotechnol 28:1057-1068 doi:10.1038/nbt.1685

31. Pradhan AD, Aday AW, Rose LM, Ridker PM (2018) Residual Inflammatory Risk on Treatment With PCSK9 Inhibition and Statin Therapy. Circulation 138:141-149 doi:10.1161/CIRCULATIONAHA.118.034645

32. Ridker PM, Devalaraja M, Baeres FMM, Engelmann MDM, Hovingh GK, Ivkovic M, Lo L, Kling D, Pergola P, Raj D, Libby P, Davidson M, Investigators R (2021) IL-6 inhibition with ziltivekimab in patients at high atherosclerotic risk (RESCUE): a double-blind, randomised, placebo-controlled, phase 2 trial. Lancet 397:2060-2069 doi:10.1016/S0140-6736(21)00520-1

33. Ridker PM, Everett BM, Thuren T, MacFadyen JG, Chang WH, Ballantyne C, Fonseca F, Nicolau J, Koenig W, Anker SD, Kastelein JJP, Cornel JH, Pais P, Pella D, Genest J, Cifkova R, Lorenzatti A, Forster T, Kobalava Z, Vida-Simiti L, Flather M, Shimokawa H, Ogawa H, Dellborg M, Rossi PRF, Troquay RPT, Libby P, Glynn RJ, Group CT (2017) Antiinflammatory Therapy with Canakinumab for Atherosclerotic Disease. N Engl J Med 377:1119-1131 doi:10.1056/NEJMoa1707914

34. Roundtree IA, Evans ME, Pan T, He C (2017) Dynamic RNA Modifications in Gene Expression Regulation. Cell 169:1187-1200 doi:10.1016/j.cell.2017.05.045

35. Shapiro MD, Fazio S (2018) Biologic bases of residual risk of cardiovascular events: A flawed concept. Eur J Prev Cardiol 25:1831-1835 doi:10.1177/2047487318798060

36. Shi H, Wei J, He C (2019) Where, When, and How: Context-Dependent Functions of RNA Methylation Writers, Readers, and Erasers. Mol Cell 74:640-650 doi:10.1016/j.molcel.2019.04.025

37. Singh RK, Haka AS, Asmal A, Barbosa-Lorenzi VC, Grosheva I, Chin HF, Xiong Y, Hla T, Maxfield FR (2020) TLR4 (Toll-Like Receptor 4)-Dependent Signaling Drives Extracellular Catabolism of LDL (Low- 
Density Lipoprotein) Aggregates. Arterioscler Thromb Vasc Biol 40:86-102 doi:10.1161/ATVBAHA.119.313200

38. Swirski FK, Libby P, Aikawa E, Alcaide P, Luscinskas FW, Weissleder R, Pittet MJ (2007) Ly-6Chi monocytes dominate hypercholesterolemia-associated monocytosis and give rise to macrophages in atheromata. J Clin Invest 117:195-205 doi:10.1172/JCl29950

39. Thygesen K, Alpert JS, Jaffe AS, Chaitman BR, Bax JJ, Morrow DA, White HD, Executive Group on behalf of the Joint European Society of Cardiology /American College of Cardiology /American Heart Association / World Heart Federation Task Force for the Universal Definition of Myocardial I (2018) Fourth Universal Definition of Myocardial Infarction (2018). Circulation 138:e618-e651 doi:10.1161/CIR.0000000000000617

40. Tyrrell DJ, Goldstein DR (2021) Ageing and atherosclerosis: vascular intrinsic and extrinsic factors and potential role of IL-6. Nat Rev Cardiol 18:58-68 doi:10.1038/s41569-020-0431-7

41. Wang X, Ji Y, Feng P, Liu R, Li G, Zheng J, Xue Y, Wei Y, Ji C, Chen D, Li J (2021) The m6A Reader IGF2BP2 Regulates Macrophage Phenotypic Activation and Inflammatory Diseases by Stabilizing TSC1 and PPARgamma. Adv Sci (Weinh) 8:2100209 doi:10.1002/advs.202100209

42. Yin $H$, Zhang $X$, Yang P, Zhang X, Peng Y, Li D, Yu Y, Wu Y, Wang Y, Zhang J, Ding X, Wang X, Yang A, Zhang R (2021) RNA m6A methylation orchestrates cancer growth and metastasis via macrophage reprogramming. Nat Commun 12:1394 doi:10.1038/s41467-021-21514-8

43. Yu R, Li Q, Feng Z, Cai L, Xu Q (2019) m6A Reader YTHDF2 Regulates LPS-Induced Inflammatory Response. Int J Mol Sci 20 doi:10.3390/ijms20061323

44. Zhang BY, Han L, Tang YF, Zhang GX, Fan XL, Zhang JJ, Xue Q, Xu ZY (2020) METTL14 regulates M6A methylation-modified primary miR-19a to promote cardiovascular endothelial cell proliferation and invasion. Eur Rev Med Pharmacol Sci 24:7015-7023 doi:10.26355/eurrev_202006_21694

45. Zhao BS, Roundtree IA, He C (2017) Post-transcriptional gene regulation by mRNA modifications. Nat Rev Mol Cell Biol 18:31-42 doi:10.1038/nrm.2016.132

\section{Figures}


a
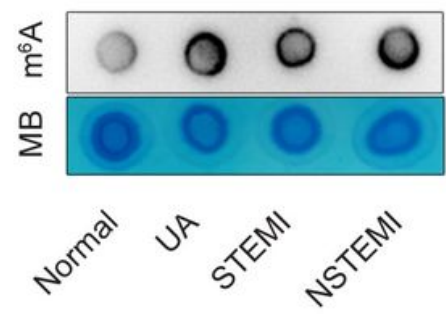

e

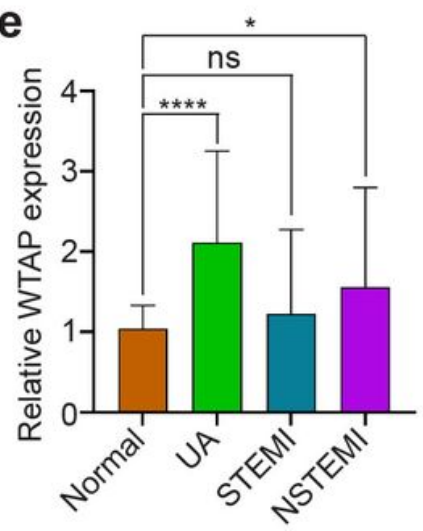

b

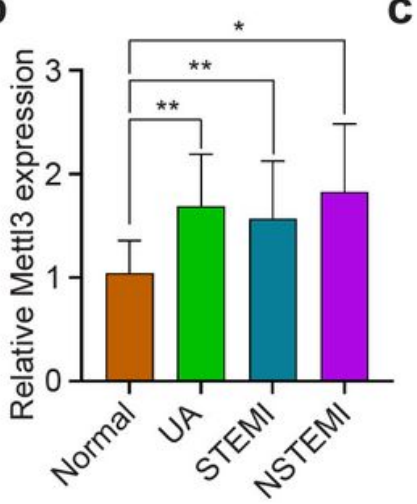

f

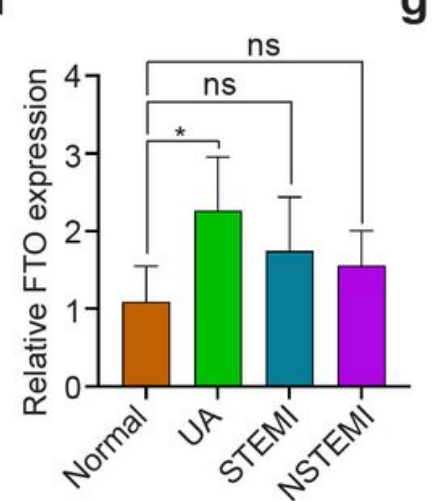

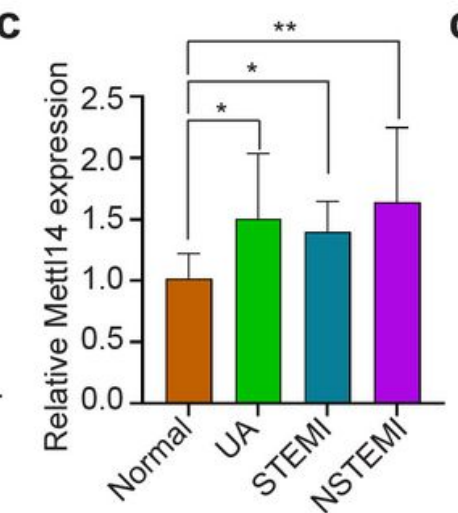

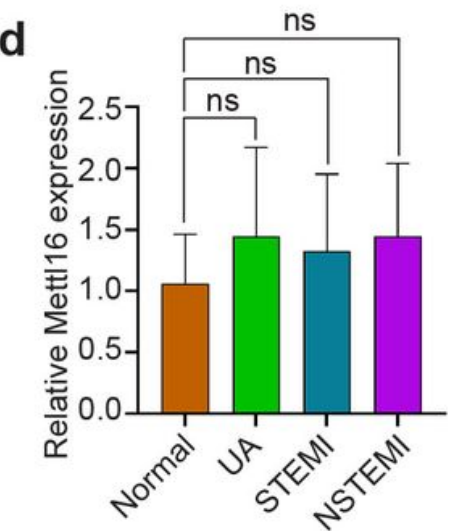

h

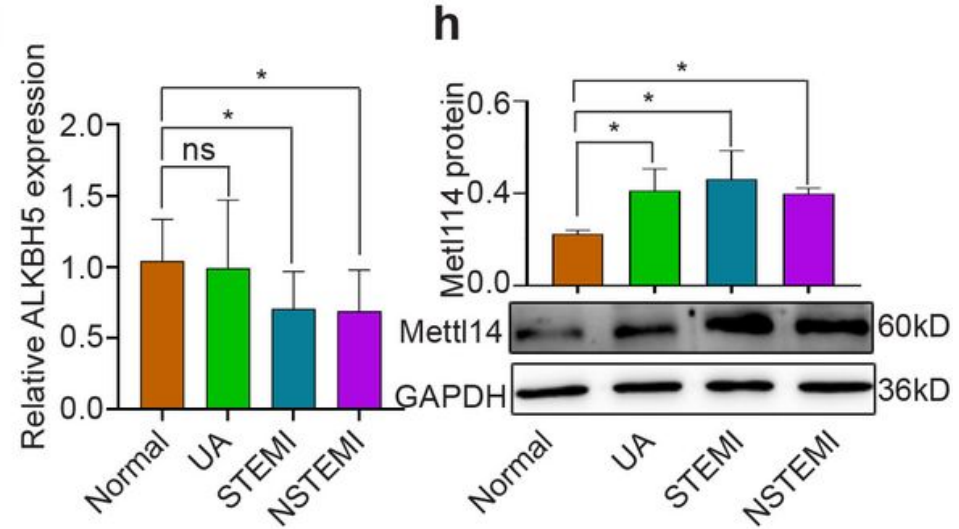

Figure 1

Expression of m6A modification methyltransferases and demethylases in coronary heart disease. a The m6A levels of PBMC RNA in CHD diseases, including UA, STEMI, and NSTEMI. mRNA levels of Mettl3 (b), Mettl14 (c), Mettl16 (d), WTAP (e), FTO (f), and ALKBH5 (g) in UA, STEMI and NSTEMI patients. Control=9 volunteers, UA=17 patients, STEMI=18 patients, NSTEMI=11 patients. The data are expressed as the mean \pm SDs. P values were determined by one-way ANOVA with Fisher's least significant difference (LSD) post-hoc test. ${ }^{*}, \mathrm{P}<0.05 ; * \star \mathrm{P}<0.01 ; * \star \star *, \mathrm{P}<0.0001$; ns, not significant. h Protein level of Mettl14 in PBMCs from patients with coronary heart disease. $n=5$ per group. The data are expressed as the means $\pm S D s$. $P$ values were determined by one-way ANOVA with Fisher's LSD post-hoc test. *, $P<0.05$. MB, methylene blue. PBMC, peripheral blood mononuclear cell. UA, unstable angina. STEMI, ST-segment elevation myocardial infarction. NSTEMI, non-ST-segment elevation myocardial infarction. 

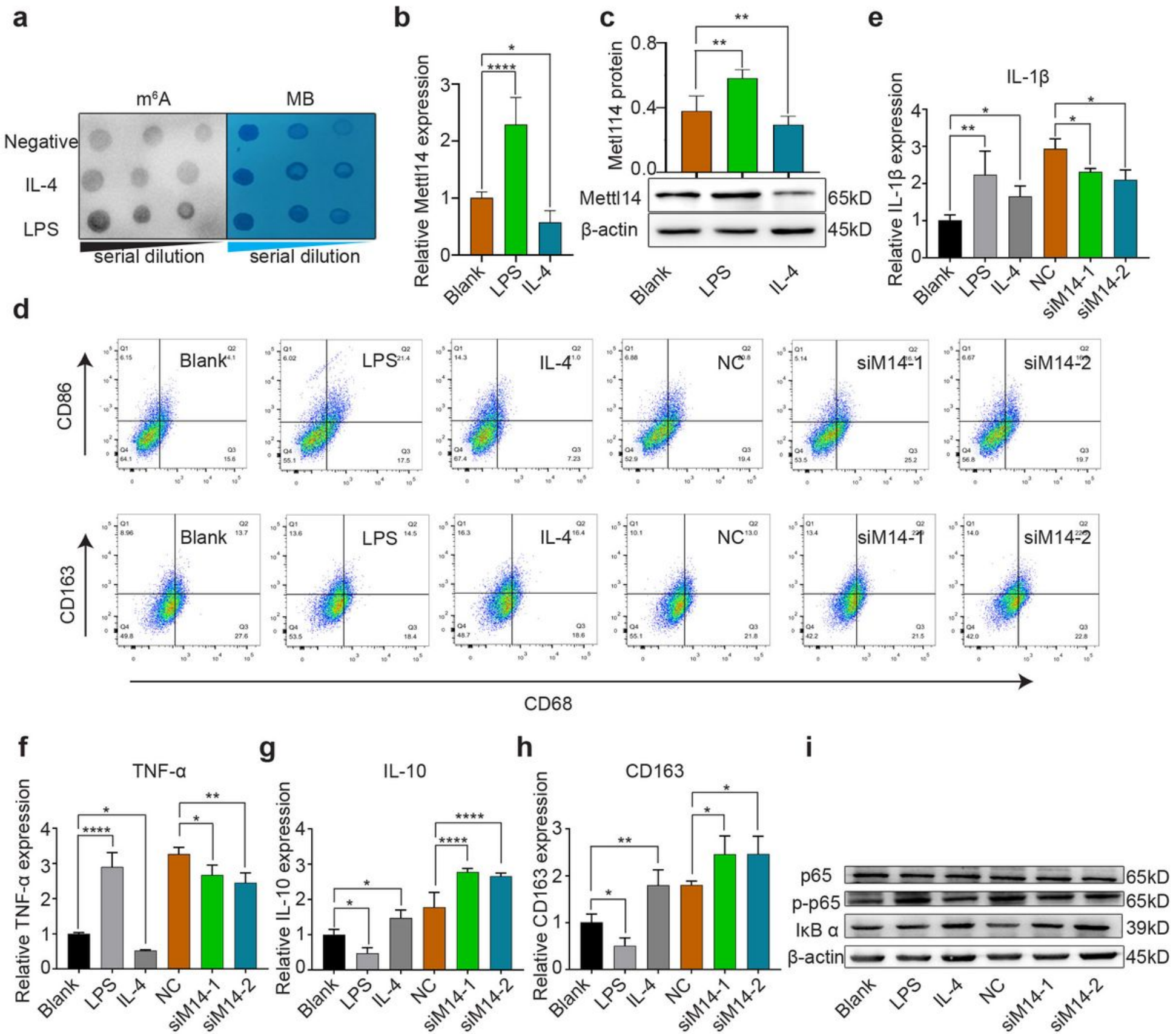

Figure 2

Knockdown of Mettl14 promotes M2 polarization of macrophages. a Dot-blot assay using an anti-m6A antibody in THP-1 cells stimulated with IL-4 $(20 \mathrm{ng} / \mathrm{ml})$ or LPS $(500 \mathrm{ng} / \mathrm{ml})$. b Mettl14 mRNA was measured by qRT-PCR in THP-1 cells stimulated with IL-4 $(20 \mathrm{ng} / \mathrm{ml})$ or LPS $(500 \mathrm{ng} / \mathrm{ml}) . \mathrm{n}=5$ per group. The data are expressed as the means \pm SDs. P values were determined by one-way ANOVA with Fisher's

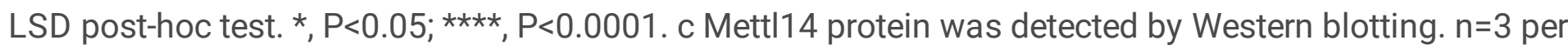
group. The data are expressed as the means \pm SDs. $P$ values were determined by one-way ANOVA with Fisher's LSD post-hoc test. ${ }^{\star \star}, \mathrm{P}<0.01$. d-i THP-1 cells treated with NC, siM14-1, or siM14-2 before treatment with IL-4 $(20 \mathrm{ng} / \mathrm{ml})$ or LPS $(500 \mathrm{ng} / \mathrm{ml})$. d The percentage of M1 polarization (CD68+CD86+) and M2 polarization (CD68+CD163+) was detected by flow cytometry. The mRNA levels of IL-1 $\beta$ (e), TNF- 
$a(f), I L-10(g)$ and CD163 (h) were analyzed by qRT-PCR. n=3 per group. The data are expressed as the means \pm SDs. P values were determined by one-way ANOVA with Fisher's LSD post-hoc test. *, $P<0.05$; **,

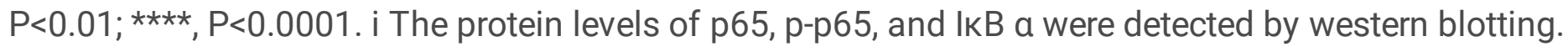
$\mathrm{MB}$, methylene blue. NC, negative control.

a

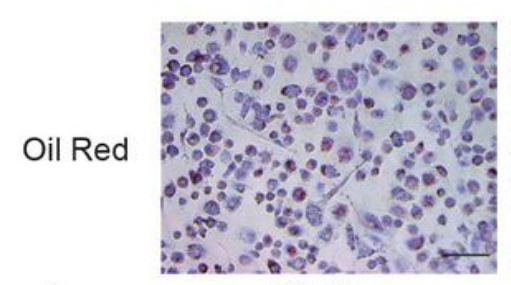

h

Oh
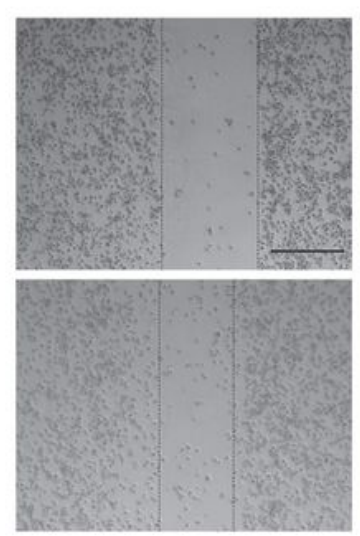

b

Blank
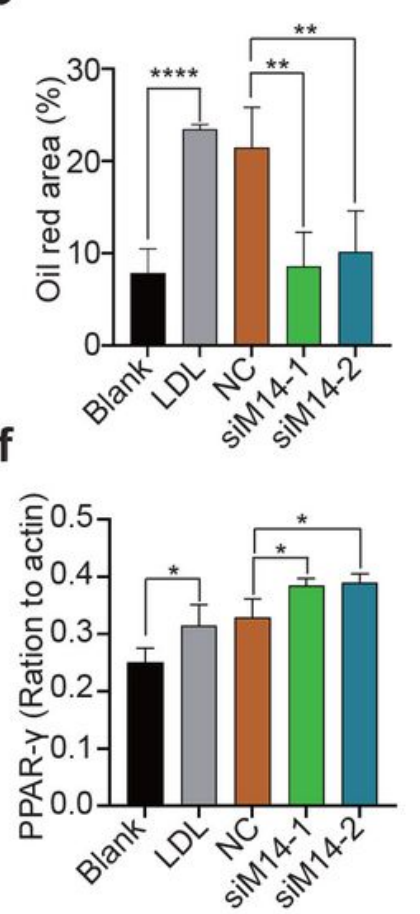

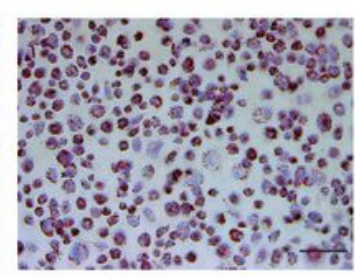

LDL
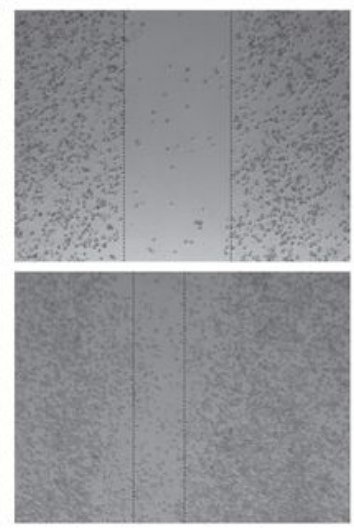

LPS

C

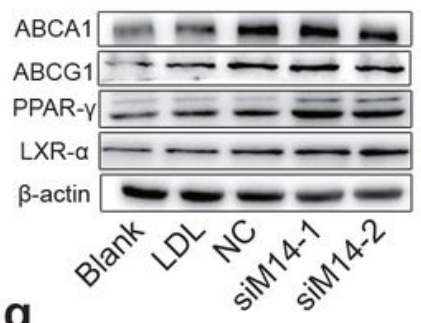

g

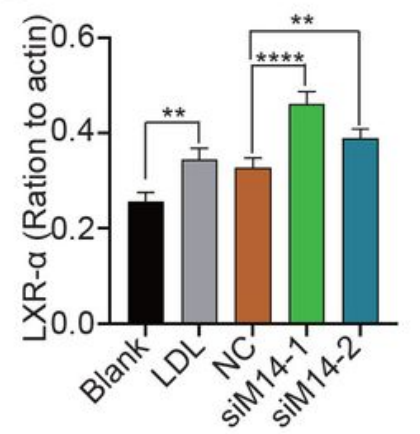

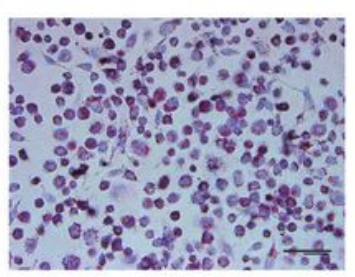

NC
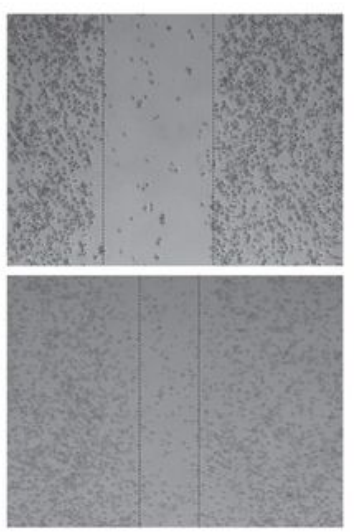

$\mathrm{NC}$

d
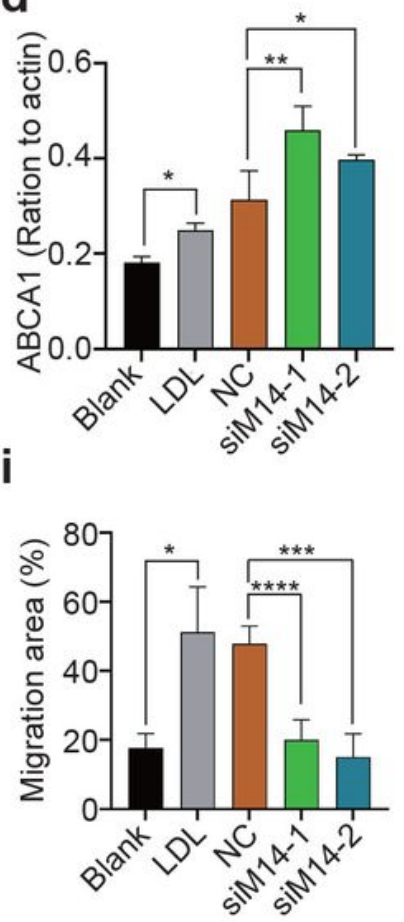

siM14-1
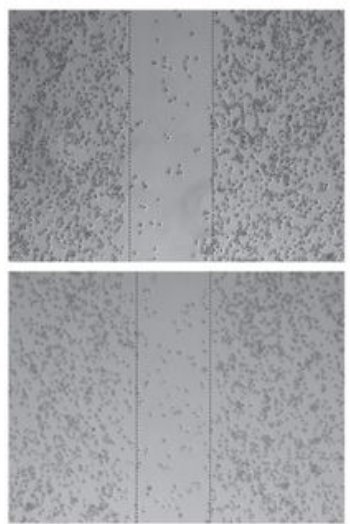

siM14-1

e

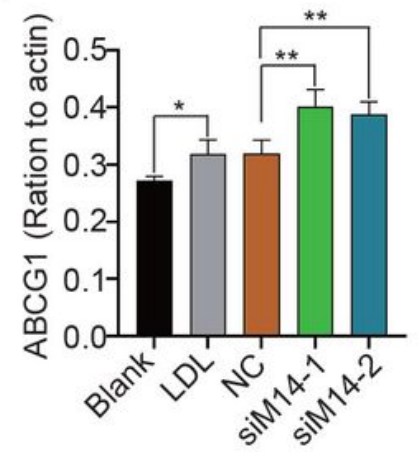

Figure 3 
Knockdown of Mettl14 inhibited foam cell formation and migration. THP-1 cells were treated with NC, siM14-1, or siM14-2 before treatment with LDL $(50 \mu \mathrm{g} / \mathrm{ml})$ or LPS $(500 \mathrm{ng} / \mathrm{ml})$. a-b Foam cell formation was assessed by oil red 0 staining. Scar bar $=100 \mu \mathrm{m}$. $n=3$ per group. The data are expressed as the means $\pm S D s$. $P$ values were determined by one-way ANOVA with Fisher's LSD post-hoc test. **, $P<0.01$; $\star \star \star \star, P<0.0001$. c-g The ABCA1, ABCG1, PPAR-y, and LXR-a protein levels were measured by western blotting. $n=3$ per group. The data are expressed as the means $\pm S D s$. $P$ values were determined by one-

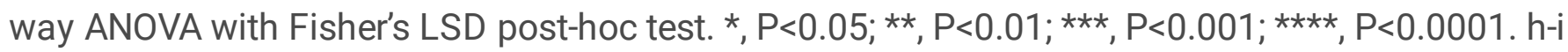
Macrophage migration was detected by wound healing assays. Scar bar $=200 \mu \mathrm{m} . \mathrm{n}=3$ per group. The data are expressed as the means \pm SDs. $P$ values were determined by one-way ANOVA with Fisher's LSD post-hoc test. *, $\mathrm{P}<0.05 ; * \star \star, P<0.001, * \star \star \star, P<0.0001$. NC, negative control. 
a
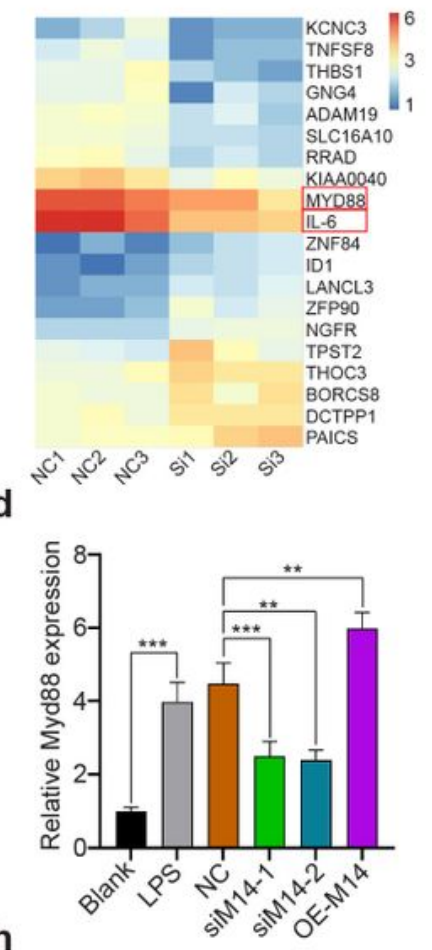

h
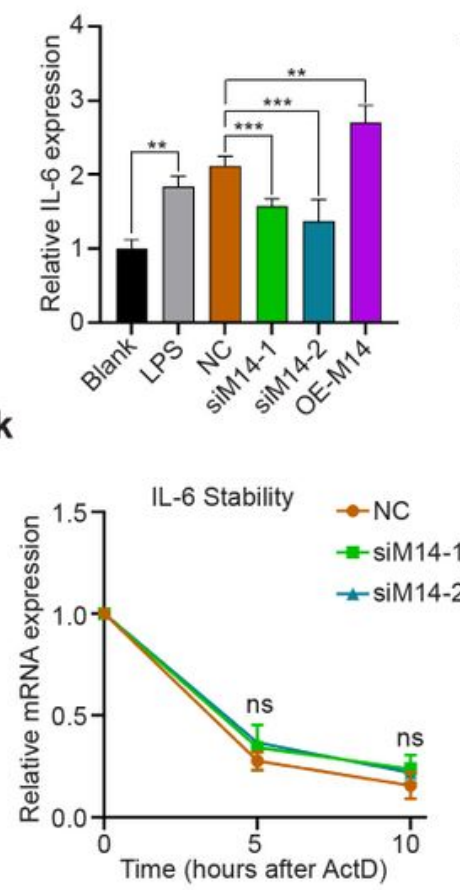

b

C
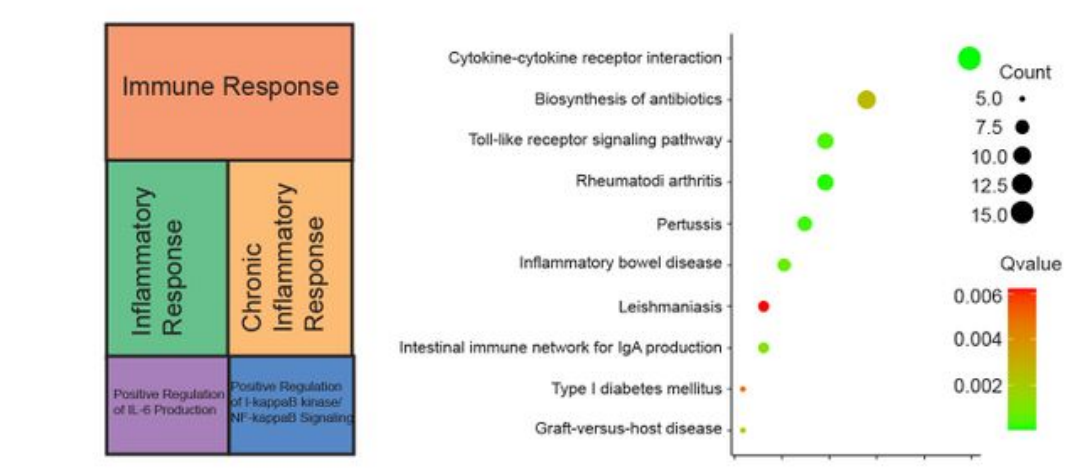

e

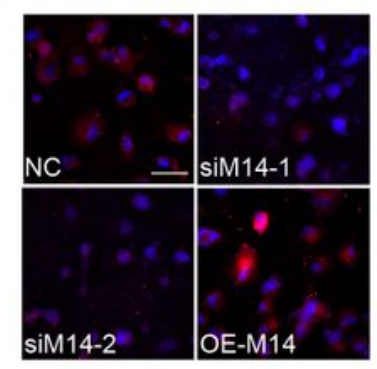

f

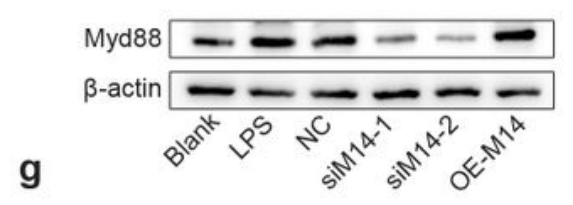

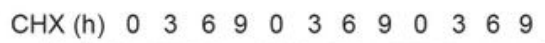
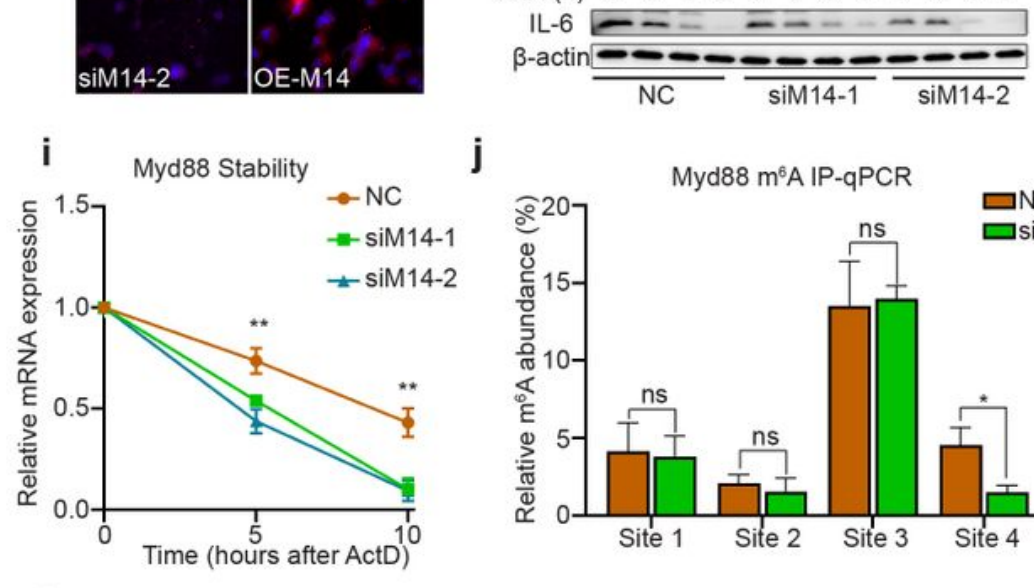

j
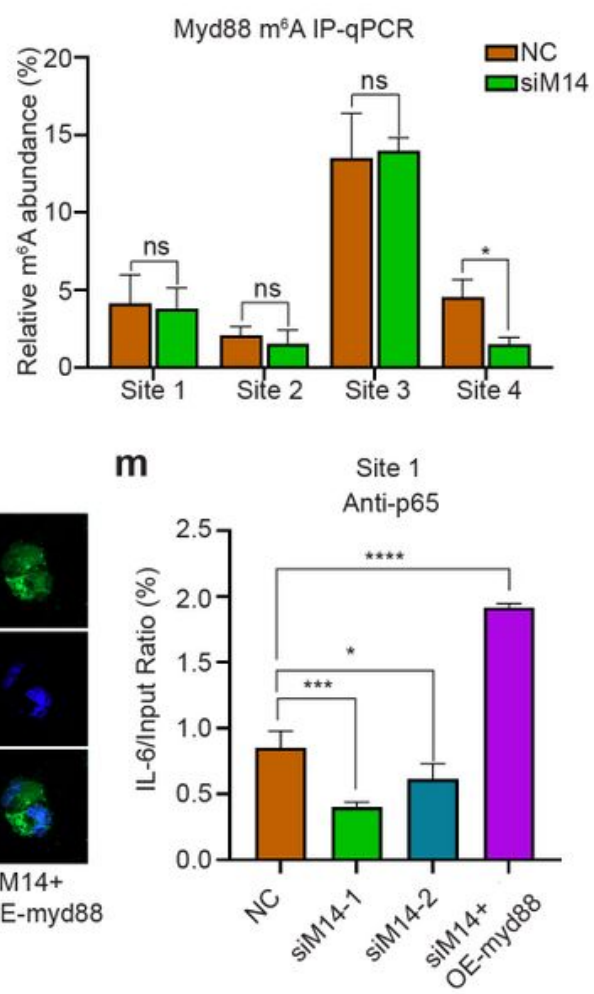

\section{Figure 4}

Mettl14 regulates Myd88 via m6A modification and IL-6 through p65. THP-1 cells were treated with NC, siM14-1, siM14-2, overexpressed (OE) Mettl14 or OE Myd88 before treatment with LPS ( $500 \mathrm{ng} / \mathrm{ml})$. a Heat map showing the DEGs in the siMettl14 group and NC group. $n=3$ per group. $b$ Results of a GO analysis performed on the DEGs. c Results of a KEGG pathway analysis performed on the DEGs. $d$ The expression of Myd88 mRNA was detected by qRT-PCR. $n=3$ per group. The data are expressed as the 
means \pm SDs. $P$ values were determined by one-way ANOVA with Fisher's LSD post-hoc test. ${ }^{*}, P<0.01$; ***, $P<0.001$. e-f Myd88 protein levels were detected by immunofluorescence (e) and western blotting (f), Scar bar $=50 \mu \mathrm{m} . . \mathrm{g}$ At $24 \mathrm{~h}$ after transfection, THP-1 cells were treated with cycloheximide (CHX). IL-6 protein was detected by western blotting. $\mathrm{h}$ IL- 6 mRNA levels was measured by qRT-PCR. $n=3$ per group. The data are expressed as the means \pm SDs. $P$ values were determined by one-way ANOVA with Fisher's LSD post-hoc test. **, $P<0.01$; $* \star \star, P<0.001$. i qRT-PCR analysis of Myd88 mRNA stability following treatment with actinomycin $D(A c t D, 2 \mu M)$. $n=3$ per group. The data are expressed as the means $\pm S D s$. $P$ values were determined by one-way ANOVA with Fisher's LSD post-hoc test. **, $P<0.01$. j RIP analysis of the interaction of m6A with Myd88 mRNA. $n=3$ per group. The data are expressed as the means \pm SDs, Student's t-test. *, P<0.05; ns, not significant. k Results of a qRT-PCR-based analysis of IL- 6 mRNA stability following treatment with actinomycin $D(A c t D, 2 \mu M)$. $n=3$ per group. The data are expressed as the means \pm SDs. P values were determined by one-way ANOVA with Fisher's LSD post-hoc test. ns, not significant. I The localization of p65 (green) was observed with immunofluorescence, and the nuclei (blue) were stained with DAPI. Scar bar $=50 \mu \mathrm{m}$. $\mathrm{m}$ ChIP assay results showing the ability of p65 proteins to bind IL -6 promoters. $n=3$ per group. The data are expressed as the means \pm SDs. $P$ values were determined by one-way ANOVA with Fisher's LSD post-hoc test. *, $P<0.05$; $* \star \star, P<0.001 ; * \star \star \star, P<0.0001$. 
a
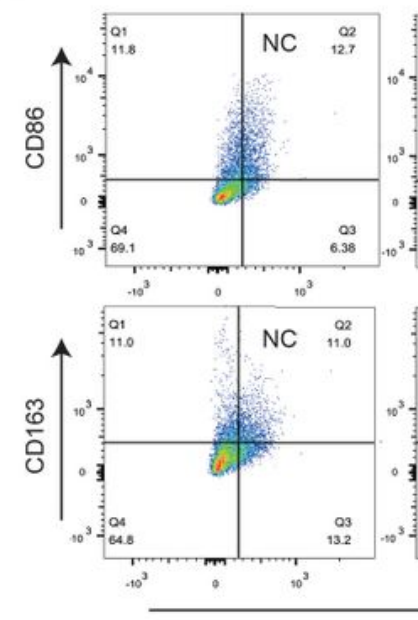

d

e
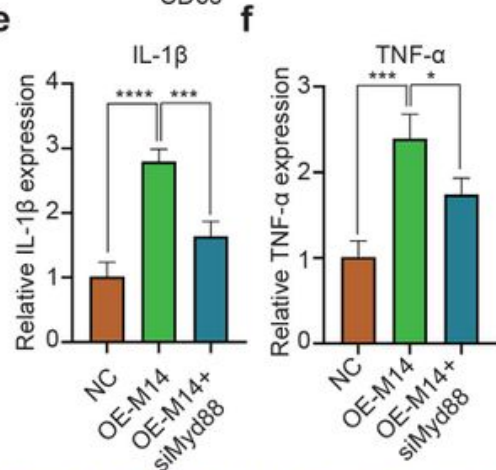

g

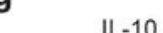

b

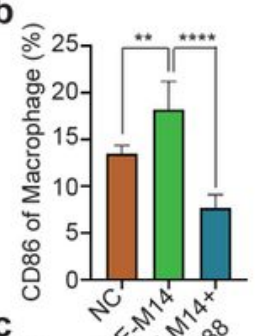

C

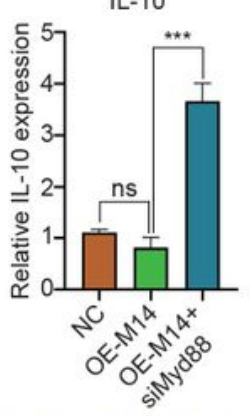

h
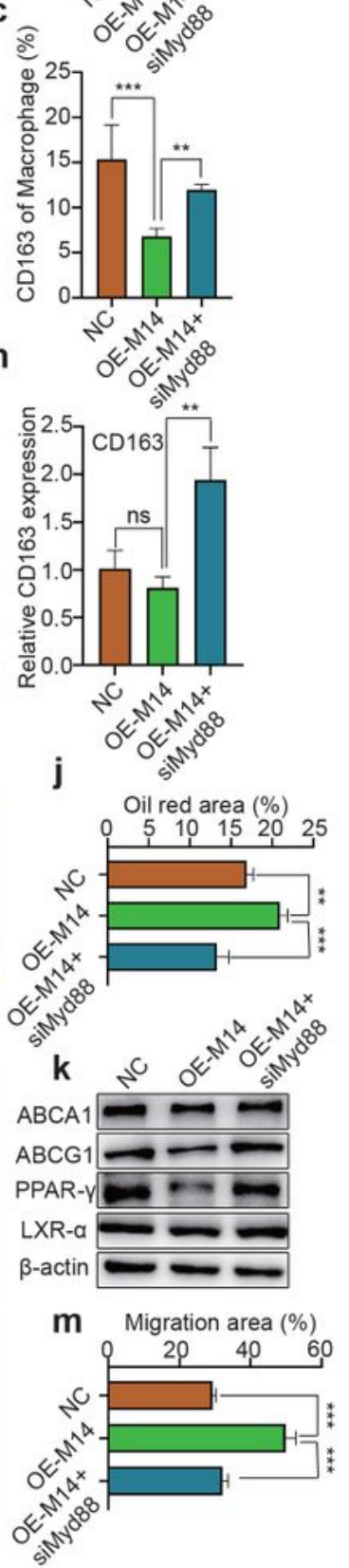

Figure 5

Mettl14 regulates the inflammatory response of macrophages through Myd88. THP-1 cells were treated with NC, OE Mettl14 or OE Mettl14 and siMyd88 before treatment with LPS (500 ng/ml). a-c The percentage of $\mathrm{M} 1$ polarization (CD68+CD86+) and M2 polarization (CD68+CD163+) was determined by flow cytometry. $n=4$ per group. The data are expressed as the means $\pm S D s$. $P$ values were determined by

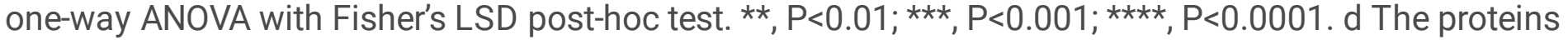


p65, p-p65 and IKB a were measured by western blotting. The mRNA levels of IL-1 $\beta$ (e), TNF-a (f), IL-10 (g) and CD163 (h) were analyzed by qRT-PCR. $n=3$ per group. The data are expressed as the means \pm SDs. $P$ values were determined by one-way ANOVA with Fisher's LSD post-hoc test. *, $P<0.05$; **, $P<0.01$; ***, $\mathrm{P}<0.001, \star \star \star \star, \mathrm{P}<0.0001$; ns, not significant. i-j Foam cell formation was detected by oil red 0 staining. Scar bar $=100 \mu \mathrm{m}$. $n=3$ per group. The data are expressed as the means \pm SDs. P values were determined by one-way ANOVA with Fisher's LSD post-hoc test. ${ }^{\star}, \mathrm{P}<0.01$, $\star \star \star, ~ P<0.001$. $k$ The ABCA1, ABCG1, PPAR$Y$, and LXR-a protein levels were measured by western blotting. I-m Macrophage migration was assessed by wound healing assays. Scar bar $=200 \mu \mathrm{m}$. $n=3$ per group. The data are expressed as the means \pm SDs. $P$ values were determined by one-way ANOVA with Fisher's LSD post-hoc test. ***, $P<0.001$. 
a

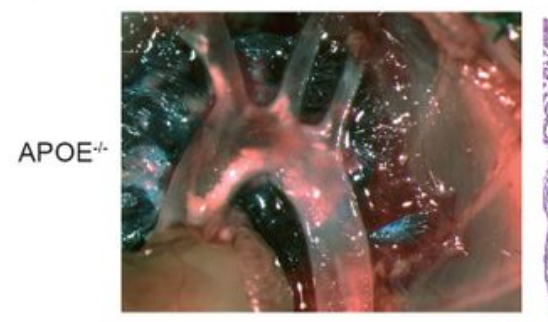

b
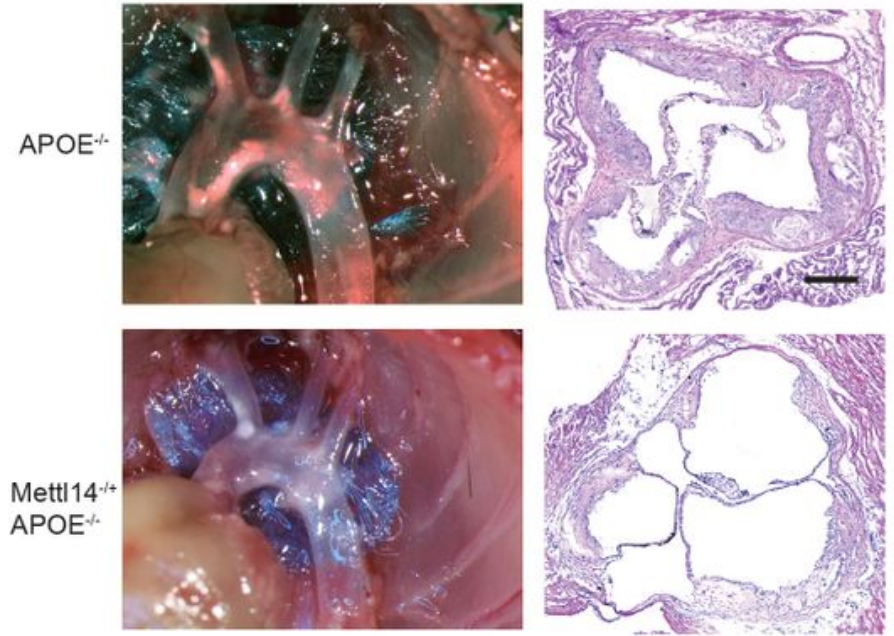

e

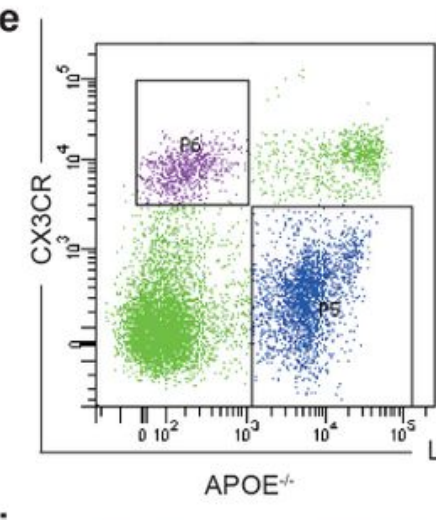

i
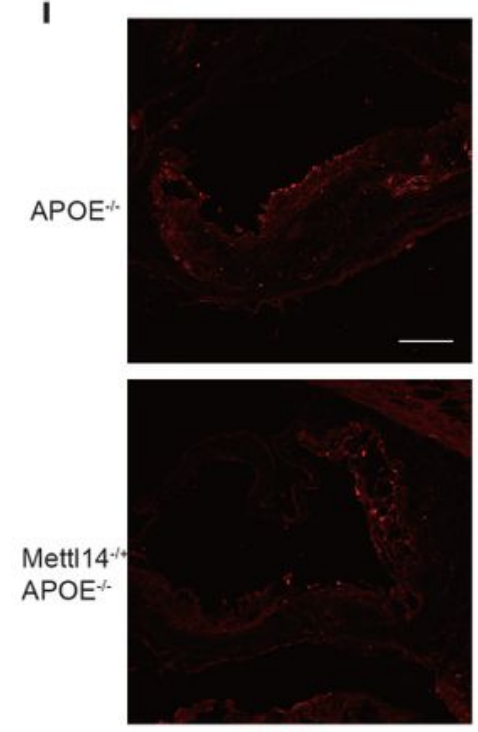

Myd88

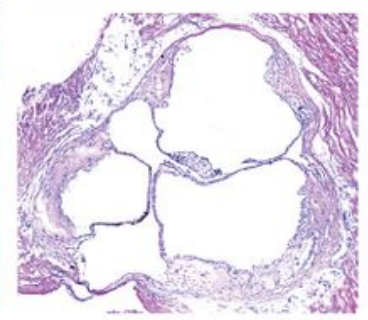

f
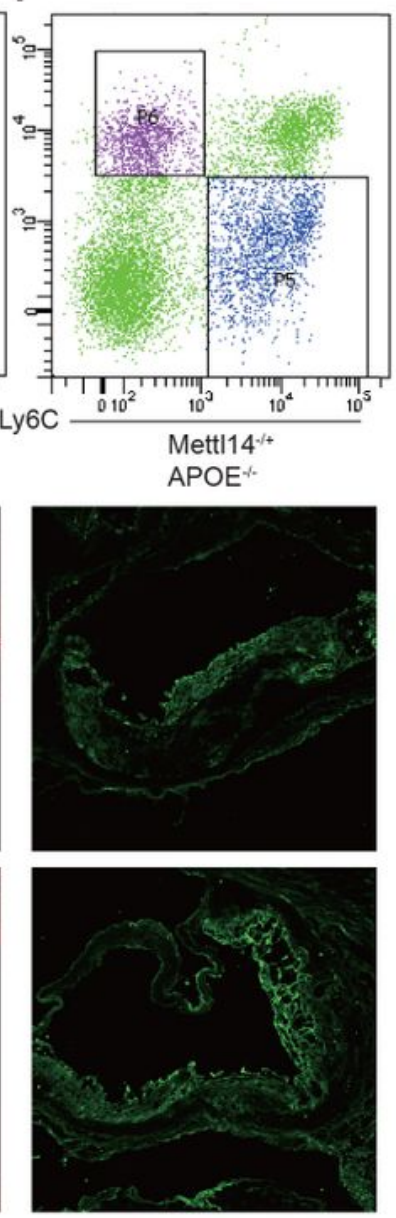

CD68 c
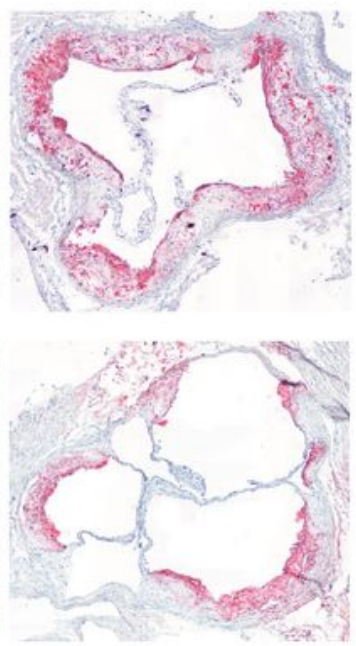

g
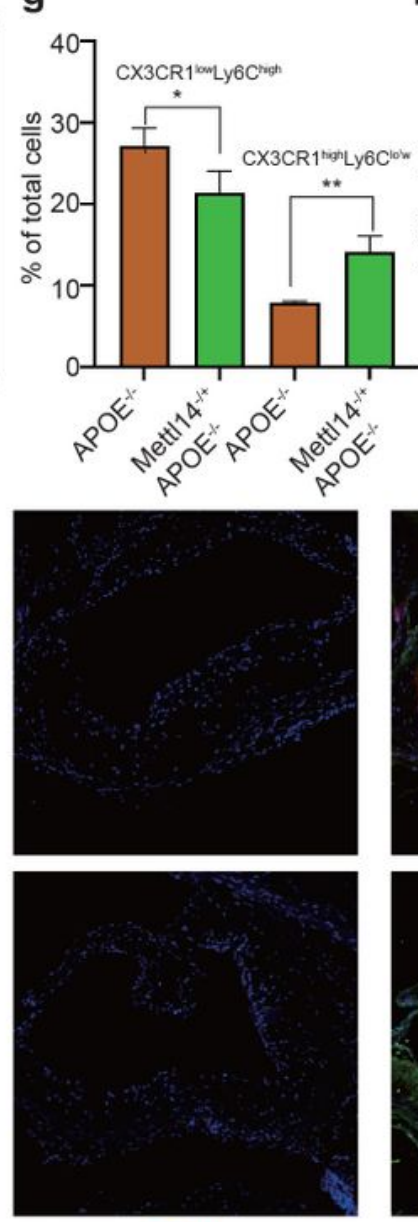

DAPI d
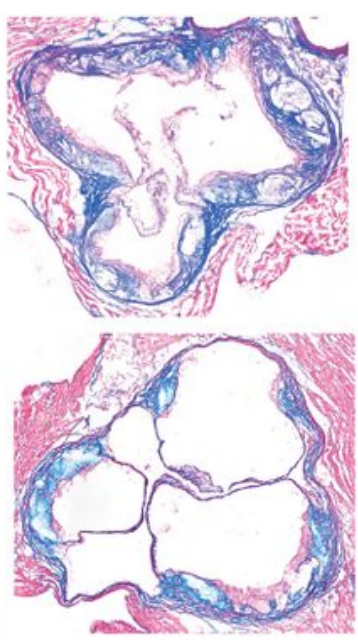

h
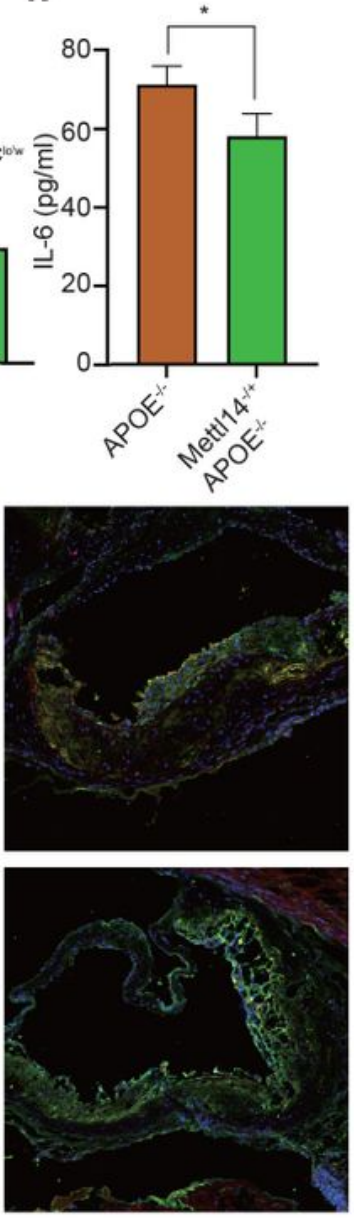

Merge

\section{Figure 6}

Mettl14 knockout mice undergo an the inflammatory response and atherosclerosis development. Mett|14-/+APOE-/- (KO) mice and APOE-/- (WT) mice were fed a high-cholesterol diet for 12 weeks. a Representative images of aortic arches are shown. Representative images of the aortic root lesion area stained with HE (b), oil red O (c) and Masson trichrome (d). Scar bar=200 $\mu \mathrm{m}$. e-g Flow cytometry analysis of monocyte populations in peripheral blood isolated from KO and WT mice. $\mathrm{n}=3$ per group. The 
data are expressed as the means \pm SDs. $P$ values were determined by student's t-test. *, $P<0.05$; $* *$, $P<0.01$. $h$ The level of IL-6 in plasma isolated from KO and WT mice. The data are expressed as the means \pm SDs. P values were determined by student's t-test. ${ }^{*}, P<0.05$. i Immunofluorescence staining showing the expression of Myd88-positive cells in atherosclerotic plaques.
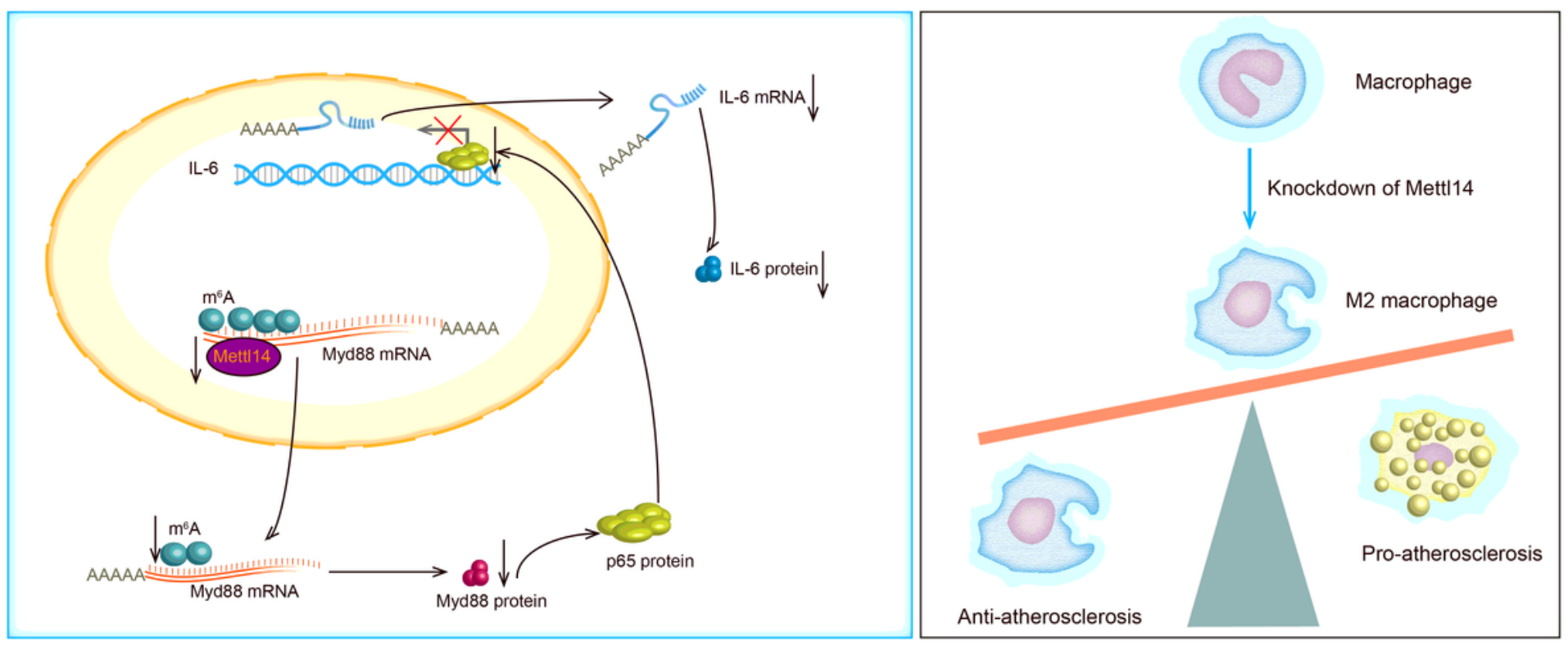

Figure 7

Graphic abstract of Mettl14 regulates the development of atherosclerosis via Myd88/IL-6 signaling pathway in macrophages.

\section{Supplementary Files}

This is a list of supplementary files associated with this preprint. Click to download.

- FigureS1.pdf

- Figures2.pdf

- FigureS3.pdf

- FigureS4.pdf

- TableS1.doc

- Tables2.xls 